\title{
CONGRUENCES OF THE CARDINALITIES OF RATIONAL POINTS OF LOG FANO VARIETIES AND LOG CALABI-YAU VARIETIES OVER THE LOG POINTS OF FINITE FIELDS
}

\section{YUKIYOSHI NAKKAJIMA}

Department of Mathematics

Tokyo Denki University

5 Asahi-Cho Senju Adachi-Ku

Tokyo 120-8551

Japan

e-mail: nakayuki@cck.dendai.ac.jp

\begin{abstract}
In this article, we give the definitions of log Fano varieties and log Calabi-Yau varieties in the framework of theory of log schemes of Fontaine-Illusie-Kato and give congruences of the cardinalities of rational points of them over the log points of finite fields.
\end{abstract}

\section{Introduction}

In this article, we discuss a new topic-rational points of the underlying schemes of log schemes in the sense of Fontaine-Illusie-Kato over the log point of a finite field-for interesting log schemes. First let us recall results on rational points of (proper smooth) schemes over a finite field.

2010 Mathematics Subject Classification: 14F30, 14F40, 14J32.

Keywords and phrases: rational points in finite fields, log varieties, Lefschetz trace formula. The author is supported from JSPS Grant-in-Aid for Scientific Research (C) (Grant No. 80287440).

Received July 11, 2019

(ㄷ) 2019 Scientific Advances Publishers 
The following is famous Ax' and Katz' theorem:

Theorem 1.1 ([1], [33]). Let $\mathbb{F}_{q}$ be the finite field with $q=p^{e}$ elements, where $p$ is a prime number. Let $n$ and $r$ be positive integers. Let $D_{i}(1 \leq i \leq r)$ be a hypersurface of $\mathbb{P}_{\mathbb{F}_{q}}^{n}$ of degree $d_{i}$. If $\sum_{i=1}^{r} d_{i} \leq n$, then $\#\left(\bigcap_{i=1}^{r} D_{i}\right)\left(\mathbb{F}_{q^{k}}\right) \equiv 1 \bmod q^{k}$

In [16], Esnault has proved the following theorem generalizing this theorem in the case where $\bigcap_{i=1}^{r} D_{i}$ is smooth over $\mathbb{F}_{q}$ and geometrically connected:

Theorem 1.2 ([16], Corollary 1.3). Let $X$ be a geometrically connected projective smooth scheme over $\mathbb{F}_{q}$. If $X / \mathbb{F}_{q}$ is a Fano variety (i.e., the inverse of the canonical sheaf $\omega_{X / \mathbb{F}_{q}}^{-1}$ of $X / \mathbb{F}_{q}$ is ample), then $\# X\left(\mathbb{F}_{q^{k}}\right) \equiv 1 \bmod q^{k}\left(k \in \mathbb{Z}_{\geq 1}\right)$

In [34], Kim has proved the following theorem and he has reproved Esnault's theorem as a corollary of his theorem by using the Lefschetz trace formula for the crystalline cohomology of $X / \mathbb{F}_{q}$ :

Theorem 1.3 ([34], Theorem 1). Let $\kappa$ be a perfect field of characteristic $p>0$. Set $\mathcal{W}:=\mathcal{W}(\kappa)$ and $K_{0}:=\operatorname{Frac}(\mathcal{W})$. Let $X$ be a projective smooth scheme over $\kappa$. If $X / \kappa$ is a Fano variety, then $H^{i}\left(X, \mathcal{W}\left(\mathcal{O}_{X}\right)\right) \otimes_{\mathcal{W}} K_{0}=0$ for $i>0$.

In [24], Gongyo et al. have proved the following theorem generalizing (1.2) for the 3-dimensional case by using methods of MMP(=minimal model program) in characteristic $p \geq 7$ : 
Theorem 1.4 ([24], Theorems 1.2, 1.3). Let $\kappa$ be as in Theorem 1.3. Assume that $p \geq 7$. Let $X$ be a geometrically connected proper variety over $\kappa$. Let $\Delta$ be an effective $\mathbb{Q}$-Cartier divisor on $X$. Assume that $(X, \Delta)$ is $k l t\left(=\right.$ Kawamata log terminal) pair over $\kappa$ and that $-\left(K_{X}+\Delta\right)$ is a $\mathbb{Q}$-Cartier ample divisor on $X$, where $K_{X}$ is the canonical divisor on $X$. Then the following hold:

(1) $H^{i}\left(X, \mathcal{W}\left(\mathcal{O}_{X}\right)\right) \otimes_{\mathcal{W}} K_{0}=0$ for $i>0$.

(2) Assume that $\kappa=\mathbb{F}_{q}$. Then $\# X\left(\mathbb{F}_{q^{k}}\right) \equiv 1 \bmod q^{k}\left(k \in \mathbb{Z}_{\geq 1}\right)$.

See [54] for the case where $-\left(K_{X}+\Delta\right)$ is nef and big and $(X, \Delta)$ is $\log$ canonical.

In this article, we give other generalizations of the Theorems 1.2 and 1.3 under the assumption of certain finiteness: we give the definition of a log Fano variety and we prove a log and stronger version (Theorem 1.5) below of Kim's theorem under the assumption as a really immediate good application of a recent result: Nakkajima-Yobuko's Kodaira vanishing theorem for a quasi- $F$-split projective log smooth scheme of vertical type ([55]). In this vanishing theorem, we use theory of log structures due to Fontaine-Illusie-Kato ([31, 32]) essentially. (See Section 3 for the precise statement of this vanishing theorem.) As a corollary of Theorem 1.5, we obtain the congruence of the cardinality of rational points of a log Fano variety over the log point of $\mathbb{F}_{q}$ (Corollary 1.6 below).

To state our result (Theorem 1.5), we first recall the notion of the quasi-Frobenius splitting height due to Yobuko, which plays an important role for log Fano varieties in this article. 
Let $Y$ be a scheme of characteristic $p>0$. Let $F_{Y}: Y \rightarrow Y$ be the Frobenius endomorphism of $Y$. Set $F:=\mathcal{W}_{n}\left(F_{Y}^{*}\right): \mathcal{W}_{n}\left(\mathcal{O}_{Y}\right) \rightarrow F_{Y *}$ $\left(\mathcal{W}_{n}\left(\mathcal{O}_{Y}\right)\right)$. This is a morphism of $\mathcal{W}_{n}\left(\mathcal{O}_{Y}\right)$-modules. In [69], Yobuko has introduced the notion of the quasi-Frobenius splitting height $h^{F}(Y)$ for $Y$. (In [loc. cit.] he has denoted it by $\mathrm{ht}^{S}(Y)$.) It is the minimum of positive integers $n$ 's such that there exists a morphism $\rho: F_{Y *}\left(\mathcal{W}_{n}\left(\mathcal{O}_{Y}\right)\right)$ $\rightarrow \mathcal{O}_{Y}$ of $\mathcal{W}_{n}\left(\mathcal{O}_{Y}\right)$-modules such that $\rho \circ F: \mathcal{W}_{n}\left(\mathcal{O}_{Y}\right) \rightarrow \mathcal{O}_{Y}$ is the natural projection. (If there does not exist such $n$, then we set $h^{F}(Y)=\infty$.) This is a highly nontrivial generalization of the notion of the Frobenius splitting by Mehta and Ramanathan in [44] because they have said that, for a scheme $Z$ of characteristic $p>0, Z$ is a Frobenius splitting(=F-split) scheme if $F: \mathcal{O}_{Z} \rightarrow F_{Z *}\left(\mathcal{O}_{Z}\right)$ has a section of $\mathcal{O}_{Z}$-modules. Because the terminology "quasi Frobenius splitting height" is too long, we call this Yobuko height.

Let $\kappa$ be a perfect field of characteristic $p>0$. Let $s$ be a log scheme whose underlying scheme is $\operatorname{Spec}(\kappa)$ and whose log structure is associated to a morphism $\mathbb{N} \ni 1 \mapsto a \in \kappa$ for some $a \in \kappa$. That is, $s$ is the log point of $\kappa$ or $\left(\operatorname{Spec}(\kappa), \kappa^{*}\right)$. Let $X / s$ be a proper (not necessarily projective) log smooth scheme of pure dimension $d$ of vertical type with $\log$ structure $\left(M_{X}, \alpha: M_{X} \rightarrow \mathcal{O}_{X}\right)$. Here "vertical type" means that $\alpha\left(\mathcal{I}_{X / s}\right) \mathcal{O}_{X}=\mathcal{O}_{X}$, where $\mathcal{I}_{X / s}$ is Tsuji's ideal sheaf of the log structure $M_{X}$ of $X$ defined in [66] and denoted by $I_{f}$ in [loc. cit.], where $f: X \rightarrow s$ is the structural morphism. (In Section 3 below we recall the definition of $\mathcal{I}_{X / s}$.) For example, the product of (locally) simple normal crossing log schemes over $s$ defined in [46], [55] and [51] is of vertical type. Let $\stackrel{\circ}{X}$ be the underlying scheme of $X$. Let $\Omega_{X / s}^{i}$ be the sheaf of log 
differential forms of degree $i$ on $\stackrel{\circ}{X}$, which has been denoted by $\omega_{X / s}^{i}$ in [31]. Set $\omega_{X / s}:=\Omega_{X / s}^{d}$. We say that $X / s$ is a log Fano scheme if $\omega_{X / s}^{-1}$ is ample. Moreover, if $\stackrel{\circ}{X}$ is geometrically connected, then we say that $X / s$ a $\log$ Fano variety.

In this article we prove the following:

Theorem 1.5. Let $X / \mathrm{s}$ be a log Fano scheme. Assume that $h^{F}(\stackrel{\circ}{X})<\infty$. Then $H^{i}\left(X, \mathcal{W}_{n}\left(\mathcal{O}_{X}\right)\right)=0$ for $i>0$ and for $n>0$. Consequently, $H^{i}\left(X, \mathcal{W}\left(\mathcal{O}_{X}\right)\right)=0$ for $i>0$.

As mentioned above, we obtain this theorem immediately by using Nakkajima-Yobuko's Kodaira vanishing theorem for a quasi- $F$-split projective log smooth scheme of vertical type ([55]). As a corollary of this theorem, we obtain the following:

Corollary 1.6. Let $X / s$ be a $\log$ Fano variety. Assume that $\kappa=\mathbb{F}_{q}$ and that $h^{F}(\stackrel{\circ}{X})<\infty$. Then

$$
\# \stackrel{\circ}{X}\left(\mathbb{F}_{q^{k}}\right) \equiv 1 \bmod q^{k} \quad\left(k \in \mathbb{Z}_{\geq 1}\right)
$$

In particular $\stackrel{\circ}{X}\left(\mathbb{F}_{q}\right) \neq \emptyset$.

This is a generalization of Esnault's Theorem 1.2 under the assumption of the finiteness of the Yobuko height. To derive Corollary 1.6 from Theorem 1.5, we use

(A) Étess-Le Stum's Lefschetz trace formula for rigid cohomology (with compact support) ([17]). 
(B) Berthelot-Bloch-Esnault's calculation of the slope < 1-part of the rigid cohomology (with compact support) via Witt sheaves ([4]) as in [5], [24] and [54]. However our proofs of Theorem 1.5 and Corollary 1.6 are very different from Esnault's, Kim's and Gongyo-Nakamura-Tanaka's proofs of Theorems 1.2, 1.3, and 1.4 in their articles because we do not use the rational connectedness of a Fano variety which has been used in them.

We guess that the assumption of the finiteness of the Yobuko height is not a strong one for log Fano schemes. However, this assumption is not always satisfied for smooth Fano schemes because the Kodaira vanishing holds if the Yobuko height is finite and because the Kodaira vanishing does not hold for certain Fano varieties ([27, 39, 65]); the Yobuko heights of them are infinity. Hence to calculate the Yobuko heights of (log) Fano schemes is a very interesting problem.

The conclusion of Corollary 1.6 holds for a proper scheme $Y / \mathbb{F}_{q}$ such that $H^{i}\left(Y, \mathcal{O}_{Y}\right)=0(i>0)$. Tanaka has kindly told me that it is not known whether there exists an example of a smooth Fano variety over $\kappa$ for which this vanishing of the cohomologies does not hold. (In [30], Joshi has already pointed out this; Shepherd-Barron has already proved that this vanishing holds for a smooth Fano variety of dimension 3 $([59,(1.5)]))$.

On the other hand, it is not clear at all that there is a precise rule as above about congruences of the cardinalities of the rational points of varieties except Fano varieties. One may think that there is no rule for them. In this article, we show that this is not the case for log Calabi-Yau varieties over $s$ of any dimension when $\stackrel{\circ}{s}=\operatorname{Spec}\left(\mathbb{F}_{q}\right)$; we are more interested in the cardinalities of the rational points of log Calabi-Yau varieties than those of log Fano varieties.

First let us recall the following suggestive observation, which seems well-known ([3]). 
Let $E$ be an elliptic curve over $\mathbb{F}_{p}$. It is well-known that $E$ is nonordinary if and only if

$$
\# E\left(\mathbb{F}_{p}\right)=p+1
$$

if $p \geq 5$. By the purity of the weight for $E / \mathbb{F}_{p}$ :

$$
\left|\# E\left(\mathbb{F}_{p}\right)-(p+1)\right| \leq 2 \sqrt{p}
$$

this equality is equivalent to a congruence

$$
\# E\left(\mathbb{F}_{p}\right) \equiv 1 \bmod p
$$

since $\sqrt{p}>2$.

In this article, we generalize the congruence (1.6.4) for higher dimensional (log) varieties as follows. (We also generalize (1.6.2) for any nonordinary elliptic curve over $\mathbb{F}_{q}$ when $p \geq 5$.)

Let $X / s$ be a proper (not necessarily projective) simple normal crossing log scheme of pure dimension $d$. Recall that, in [55], we have said that $X / s$ is a log Calabi-Yau scheme of pure dimension $d$ if $H^{i}\left(X, \mathcal{O}_{X}\right)=0(0<i<d)$ and $\omega_{X / s} \simeq \mathcal{O}_{X}$. Moreover, if $\stackrel{\circ}{X}$ is geometrically connected, then we say that $X / s$ is a log Calabi-Yau variety of pure dimension $d$. (This is a generalization of a log K3 surface defined in [46].) Note that $H^{d}\left(X, \mathcal{O}_{X}\right)=H^{d}\left(X, \omega_{X / s}\right) \simeq \kappa$. The last isomorphism is obtained by log Serre duality of Tsuji ([66, (2.21)]). More generally, we consider a proper scheme $Y$ of pure dimension $d$ satisfying only the following four conditions:

(a) $H^{0}\left(Y, \mathcal{O}_{Y}\right)=\kappa$,

(b) $H^{i}\left(Y, \mathcal{W}\left(\mathcal{O}_{Y}\right)\right)_{K_{0}}=0$ for $0<i<d-1$,

(c) $H^{d-1}\left(Y, \mathcal{O}_{Y}\right)=0$ if $d \geq 2$,

(d) $H^{d}\left(Y, \mathcal{O}_{Y}\right) \simeq \kappa$. 
Let $\Phi_{Y / \kappa}$ be the Artin-Mazur formal group of $Y / \kappa$ in degree $d$, that is, $\Phi_{Y / \kappa}$ is the following functor:

$$
\Phi_{Y / \kappa}(A):=\Phi_{Y / \kappa}^{d}(A):=\operatorname{Ker}\left(H_{\mathrm{et}}^{d}\left(Y \otimes_{\kappa} A, \mathbb{G}_{m}\right) \rightarrow H_{\mathrm{et}}^{d}\left(Y, \mathbb{G}_{m}\right)\right) \in(\mathrm{Ab}),
$$

for artinian local $\kappa$-algebras $A$ 's with residue fields $\kappa$. Then $\Phi_{Y / \kappa}$ is prorepresented by a commutative formal Lie group over $\kappa$ ([2]). Denote the height of $\Phi_{Y / \kappa}$ by $h(Y / \kappa)$. We prove the following:

Theorem 1.7. Let $Y / \kappa$ be as above. Assume that $\kappa=\mathbb{F}_{q}$. Set $h:=h\left(Y / \mathbb{F}_{q}\right)$. Then the following hold:

(1) Assume that $h=\infty$. Then

$$
\# Y\left(\mathbb{F}_{q^{k}}\right) \equiv 1 \bmod q^{k} \quad\left(k \in \mathbb{Z}_{\geq 1}\right)
$$

In particular, $Y\left(\mathbb{F}_{q}\right) \neq \emptyset$.

(2) Assume that $2 \leq h<\infty$. Let \lceil\rceil be the ceiling function: $\lceil x\rceil:=\min \{n \in \mathbb{Z} \mid x \leq n\}$. Then

$$
\# Y\left(\mathbb{F}_{q^{k}}\right) \equiv 1 \bmod p^{\left\lceil e k\left(1-h^{-1}\right)\right\rceil} \quad\left(k \in \mathbb{Z}_{\geq 1}\right) .
$$

In particular, $Y\left(\mathbb{F}_{q}\right) \neq \emptyset\left(\right.$ recall that $\left.e=\log _{p} q\right)$.

(3) Assume that $h=1$. Then

$$
\# Y\left(\mathbb{F}_{q^{k}}\right) \not \equiv 1 \bmod p \quad\left(k \in \mathbb{Z}_{\geq 1}\right) .
$$

(In particular $Y\left(\mathbb{F}_{q^{k}}\right)$ can be empty.)

To give the statement (1.7) is a nontrivial work. However the proof of Theorem 1.7 is not difficult. (It does not matter whether the proof is not difficult.) As far as we know, Theorem 1.7 even in the 2-dimensional trivial logarithmic and smooth case, i.e., the case of K3 surfaces over finite fields, is a new result. Even in the case $d=1, Y$ need not be assumed to be an elliptic curve over $\mathbb{F}_{q}$. 
The heights of Artin-Mazur formal groups describe the different phenomena about the congruences of rational points for schemes satisfying four conditions (a), (b), (c), and (d).

By using Theorem 1.7, we raise an important problem how the certain supersingular prime ideals are distributed for a smooth CalabiYau variety of dimension less than or equal to 2 over a number field. (I think that this problem has no relation with Sato-Tate conjecture in non-CM cases.)

To obtain Theorem 1.7, we use the Theorems (A) and (B) explained after Corollary 1.6 again and the determination of the slopes of the Dieudonné module $D\left(\Phi_{Y / \kappa}\right)$ of $\Phi_{Y / \kappa}$.

Theorem 1.7 (3) also follows from a formula of ([7], (3.7.2)) immediately.

The contents of this article are as follows.

In Section 2, we recall Étess-Le Stum's Lefschetz trace formula for rigid cohomology, Berthelot-Bloch-Esnault's theorem and the congruence of the cardinality of rational points of a separated scheme of finite type over a finite field.

In Section 3, we prove Theorem 1.5 and Corollary 1.6.

In Section 4, we prove (1.7). We also raise the important problem about the distribution of supersingular primes already mentioned.

In Section 5, we give the formulas of two kinds of zeta functions of a few projective SNCL(=simple normal crossing log) schemes over the log point of a finite field. One kind of them gives us examples of the conclusions of the congruences in Corollary 1.6 and Theorem 1.7.

In Section 6, we give a remark on Van der Geer and Katsura's characterization of the height $h(Y / \kappa)$ ([67]). 
Notations: (1) For an element $a$ of a commutative ring $A$ with unit element and for an $A$-modules $M, M / a$ denotes $M / a M$.

(2) For a finite field $\mathbb{F}_{q}, s_{\mathbb{F}_{q}}$ denotes the log point whose underlying scheme is $\operatorname{Spec}\left(\mathbb{F}_{q}\right)$.

\section{Preliminaries}

In this section, we recall Étess-Le Stum's Lefschetz trace formula for rigid cohomology with compact support ([17]) and Berthelot-BlochEsnault's calculation of the slope < 1-part of the rigid cohomology with compact support via Witt sheaves with compact support ([4]).

Let $K_{0}\left(\mathbb{F}_{q}\right)$ be the fraction field of the Witt ring $\mathcal{W}\left(\mathbb{F}_{q}\right)$ of $\mathbb{F}_{q}$. Let $Y$ be a separated scheme of finite type over $\mathbb{F}_{q}$ of dimension $d$. Let $F_{q}: Y \rightarrow Y$ be the $q$-th power Frobenius endomorphism of $Y$. The following is Étess-Le Stum's Lefschetz trace formula proved in [17, Théorème II]:

$$
\# Y\left(\mathbb{F}_{q}\right)=\sum_{i=0}^{2 d}(-1)^{i} \operatorname{Tr}\left(F_{q}^{*} \mid H_{\text {rig, } c}^{i}\left(Y / K_{0}\left(\mathbb{F}_{q}\right)\right)\right) .
$$

Let $\left\{\alpha_{i j}\right\}_{j}$ be the set of the eigenvalues of $F_{q}^{*}$ on $H_{\text {rig, } c}^{i}\left(Y / K_{0}\left(\mathbb{F}_{q}\right)\right)$. Then

$$
\# Y\left(\mathbb{F}_{q}\right)=\sum_{i=0}^{2 d}(-1)^{i}\left(\sum_{j} \alpha_{i j}\right) .
$$

By $[10,(3.1 .2)]$ (see also [49, (17.2)]),

$$
\max \{0, i-d\} \leq \operatorname{ord}_{q}\left(\alpha_{i j}\right) \leq \min \{i, d\} .
$$

Henceforth we consider the equalities (2.0.1) and (2.0.2) as the equalities in the integer ring $\overline{\mathcal{W}\left(\mathbb{F}_{q}\right)}$ of an algebraic closure $\overline{K_{0}\left(\mathbb{F}_{q}\right)}$ of $K_{0}\left(\mathbb{F}_{q}\right)$. 
Let $\kappa$ be a perfect field of characteristic $p>0$. Let $Y / \kappa$ be a separated scheme of finite type. Let $K_{0}$ be the fraction field of the Witt ring $\mathcal{W}$ of $\kappa$. Let $H_{\text {rig,c }}^{i}\left(Y / K_{0}\right)_{[0,1)}$ be the slope $<1$-part of the rigid cohomology $H_{r i g, c}^{i}\left(Y / K_{0}\right)$ with compact support with respect to the absolute Frobenius endomorphism of $Y$. Let $H_{c}^{i}\left(Y, \mathcal{W}\left(\mathcal{O}_{Y, K_{0}}\right)\right)$ be the cohomology of the Witt sheaf with compact support of $Y / K_{0}$ defined by Berthelot et al. in [4]:

$$
H_{c}^{i}\left(Y, \mathcal{W}\left(\mathcal{O}_{Y, K_{0}}\right)\right):=H^{i}\left(Y, \mathcal{W}\left(\mathcal{I}_{K_{0}}\right)\right)
$$

where $\mathcal{W}\left(\mathcal{I}_{K_{0}}\right):=\operatorname{Ker}\left(\mathcal{W}\left(\mathcal{O}_{Z}\right)_{K_{0}} \rightarrow \mathcal{W}\left(\mathcal{O}_{Z} / \mathcal{I}\right)_{K_{0}}\right)$ and $\mathcal{I}$ is a coherent ideal sheaf of $\mathcal{O}_{Z}$ for an open immersion $Y \stackrel{\subseteq}{\rightarrow} Z$ into a proper scheme over $\kappa$ such that $V(\mathcal{I})=Z \backslash Y$. They have proved that $H_{c}^{i}\left(Y, \mathcal{W}\left(\mathcal{O}_{Y, K_{0}}\right)\right)$ is independent of the choice of the closed immersion. By the definition of $H_{c}^{i}\left(Y, \mathcal{W}\left(\mathcal{O}_{Y, K_{0}}\right)\right)$, we have the following exact sequence:

$$
\begin{aligned}
& H_{c}^{i}\left(Y, \mathcal{W}\left(\mathcal{O}_{Y, K_{0}}\right)\right) \rightarrow H^{i}\left(Z, \mathcal{W}\left(\mathcal{O}_{Z, K_{0}}\right)\right) \rightarrow H^{i}\left(Z, \mathcal{W}\left(\mathcal{O}_{Z} / \mathcal{I}\right)_{K_{0}}\right) \\
& \rightarrow H_{c}^{i}\left(Y, \mathcal{W}\left(\mathcal{O}_{Y, K_{0}}\right)\right) \rightarrow \cdots
\end{aligned}
$$

By replacing $Z$ by the closure of $Y$ in $Z$, we see that

$$
H_{c}^{i}\left(Y, \mathcal{W}\left(\mathcal{O}_{Y, K_{0}}\right)\right)=0
$$

if $i>d$. Then they have proved that there exists the following contravariantly functorial isomorphism

$$
H_{\text {rig, } c}^{i}\left(Y / K_{0}\right)_{[0,1)} \stackrel{\sim}{\rightarrow} H_{c}^{i}\left(Y, \mathcal{W}\left(\mathcal{O}_{Y, K_{0}}\right)\right)
$$

([4, Theorem 1.1]). 
Now let us come back to the case $\kappa=\mathbb{F}_{q}$. Since

$$
\begin{aligned}
& H_{\text {rig }, c}^{i}\left(Y / K_{0}\left(\mathbb{F}_{q}\right)\right)=\sum_{j=0}^{d-1} H_{\text {rig, } c}^{i}\left(Y / K_{0}\left(\mathbb{F}_{q}\right)\right)_{[j, j+1)} \oplus H_{\text {rig, },}^{i}\left(Y / K_{0}\left(\mathbb{F}_{q}\right)\right)_{[d]}, \\
& \# Y\left(\mathbb{F}_{q}\right)= \sum_{i=0}^{2 d}(-1)^{i} \sum_{j=0}^{d-1} \operatorname{Tr}\left(F_{q}^{*} \mid H_{\text {rig }, c}^{i}\left(Y / K_{0}\left(\mathbb{F}_{q}\right)\right)_{[j, j+1)}\right) \\
&+\sum_{i=d}^{2 d}(-1)^{i} \operatorname{Tr}\left(F_{q}^{*} \mid H_{\text {rig }, c}^{i}\left(Y / K_{0}\left(\mathbb{F}_{q}\right)\right)_{[d]}\right)
\end{aligned}
$$

Hence we have the following congruence by (2.0.5) and (2.0.6):

$$
\# Y\left(\mathbb{F}_{q}\right) \equiv \sum_{i=0}^{d}(-1)^{i} \operatorname{Tr}\left(F_{q}^{*} \mid H_{c}^{i}\left(Y, \mathcal{W}\left(\mathcal{O}_{Y, K_{0}}\right)\right)\right) \quad \bmod q
$$

in $\overline{\mathcal{W}\left(\mathbb{F}_{q}\right)}$.

Remark 2.1. In [4, (1.4)], the following zeta function:

$$
Z^{\mathcal{W}}\left(Y / \mathbb{F}_{q}, t\right):=\prod_{i=0}^{d} \operatorname{det}\left(1-t F_{q}^{*} \mid H_{c}^{i}\left(Y, \mathcal{W}\left(\mathcal{O}_{Y}\right)\right)_{K_{0}}\right)^{(-1)^{i+1}}
$$

which is equal to the zeta function

$$
Z^{<1}\left(Y / \mathbb{F}_{q}, t\right):=\prod_{\operatorname{ord}_{q}\left(\alpha_{i j}\right)<1}\left(1-\alpha_{i j} t\right)^{(-1)^{i+1}},
$$

has been considered. In this article we do not need this zeta function. We do not need Ax's theorem in [1] (see [4, Proposition 6.3]) either.

\section{Proofs of Theorem 1.5 and Corollary 1.6}

It is well-known that the analogue of Kodaira's vanishing theorem for projective smooth schemes over a field of characteristic 0 ([35]) do not hold in characteristic $p>0$ in general ([57]). However, in [55], we have 
proved the Kodaira vanishing theorem in characteristic $p>0$ under the assumption of the finiteness of the Yobuko height. To state this theorem precisely, we recall the definition of the vertical type for a relative log scheme.

For a commutative monoid $P$ with unit element, an ideal is, by definition, a subset $I$ of $P$ such that $P I \subset I$. An ideal $\mathfrak{p}$ of $P$ is called a prime ideal if $P \backslash \mathfrak{p}$ is a submonoid of $P([32,(5.1)])$. For a prime ideal $\mathfrak{p}$ of $P$, the height $h t(\mathfrak{p})$ is the maximal length of sequence's $\mathfrak{p} \supsetneq \mathfrak{p}_{1}$ $\supsetneq \cdots \supsetneq \mathfrak{p}_{r}$ of prime ideals of $P$. Let $h: Q \rightarrow P$ be a morphism of monoids. A prime ideal $\mathfrak{p}$ of $P$ is said to be horizontal with respect to $h$ if $h(Q) \subset P \backslash \mathfrak{p}([66,(2.4)])$.

Let $Y \rightarrow Z$ be a morphism of fs(=fine and saturated) log schemes. Let $h: Q \rightarrow P$ be a local chart of $g$ such that $P$ and $Q$ are saturated. Set $I:=\{a \in P \mid a \in \mathfrak{p}$ for any horizontal prime ideal of $P$ of height 1 with respect to $h$.

Let $\mathcal{I}_{Y / Z}$ be the ideal sheaf of $M_{Y}$ generated by $\operatorname{Im}\left(I \rightarrow M_{Y}\right)$. In [66, (2.6)], Tsuji has proved that $\mathcal{I}_{Y / Z}$ is independent of the choice of the local chart $h$. Let $\mathcal{I}_{Y / Z} \mathcal{O}_{Y}$ be the ideal sheaf of $\mathcal{O}_{Y}$ generated by the image of $\mathcal{I}_{Y / Z}$.

Definition 3.1. We say that $Y / Z$ is of vertical type if $\mathcal{I}_{Y / Z} \mathcal{O}_{Y}=\mathcal{O}_{Y}$

In [55, (1.9)] we have proved the following theorem:

Theorem 3.2 (Log Kodaira Vanishing theorem). Let $Y \rightarrow s$ be a projective log smooth morphism of Cartier type of fs log schemes. Assume that $\stackrel{\circ}{Y}$ is of pure dimension d. Let $\mathcal{L}$ be an ample invertible sheaf on $\stackrel{\circ}{Y}$. Assume that $h^{F}(\stackrel{\circ}{Y})<\infty$. Then $H^{i}\left(Y, \mathcal{I}_{Y / s} \omega_{Y / s} \otimes_{\mathcal{O}_{Y}} \mathcal{L}\right)=0$ for $i>0$. In particular, if $Y / s$ is of vertical type, then $H^{i}\left(Y, \omega_{Y / s} \otimes_{\mathcal{O}_{Y}} \mathcal{L}\right)=0$ for $i>0$. 
Now let us prove Theorem 1.5 and Corollary 1.6 quickly. Let the notations be as in Corollary 1.6. Since $\omega_{X / s}^{-1}$ is ample, $H^{i}\left(X, \mathcal{O}_{X}\right)=0$ for $i>0$ by Theorem 3.2. Hence, by the following exact sequence

$$
0 \rightarrow \mathcal{W}_{n-1}\left(\mathcal{O}_{X}\right) \stackrel{V}{\rightarrow} \mathcal{W}_{n}\left(\mathcal{O}_{X}\right) \rightarrow \mathcal{O}_{X} \rightarrow 0
$$

$H^{i}\left(X, \mathcal{W}_{n}\left(\mathcal{O}_{X}\right)\right)=0$ for $i>0$ and $n>0$. Hence

$$
H^{i}\left(X, \mathcal{W}\left(\mathcal{O}_{X}\right)\right)=\underset{\leftarrow}{\lim } H^{i}\left(X, \mathcal{W}_{n}\left(\mathcal{O}_{X}\right)\right)=0
$$

Thus we have proved Theorem 1.5.

Next let us prove Corollary 1.6. It suffices to prove Corollary 1.6 for the case $k=1$ by considering the base change $X \otimes_{\mathbb{F}_{q}} \mathbb{F}_{q^{k}}$. Because $H^{0}\left(X, \mathcal{W}\left(\mathcal{O}_{X}\right)\right)=\mathcal{W}\left(\mathbb{F}_{q}\right)$ and $F_{q}^{*}=$ id on $H^{0}\left(X, \mathcal{W}\left(\mathcal{O}_{X}\right)\right)$, we obtain the following by (2.0.8):

$$
\# \stackrel{\circ}{X}\left(\mathbb{F}_{q}\right) \equiv 1 \quad \bmod \quad q
$$

in $\overline{\mathcal{W}\left(\mathbb{F}_{q}\right)}$. This shows Corollary 1.6.

Remark 3.3. (1) If $X$ is a Fano variety over $\mathbb{Q}$, then the reduction $\mathcal{X}$ $\bmod p$ of a flat model $\mathcal{X}$ over $\mathbb{Z}$ of $X$ for $p \gg 0$ is a Fano variety and $F$-split ([6, Exercise 1.6. E5]). In particular, $h^{F}(\mathcal{X} \bmod p)<\infty$ for $p \gg 0$.

(2) As pointed out in [6, p. 58], a Fano variety $X$ is not necessarily $F$-split.

The Kodaira vanishing theorem does not hold for certain Fano varieties $([27,39,65])$. By Theorem 3.2 we see that the Yobuko heights of them are infinity. 
(3) Let $X / s$ be an SNCL Fano scheme of pure dimension $d$. Then any irreducible component of $\stackrel{\circ}{X}_{i}$ of $\stackrel{\circ}{X}$ is Fano. Indeed, since $\omega_{X / s}^{-1}$ is ample, $\omega_{X / s}^{-1} \otimes_{\mathcal{O}_{X}} \mathcal{O}_{X_{i}}=\underset{X_{i} / \kappa}{-1}\left(-\sum_{j} \log D_{j}\right)$ is also ample. Here $\left\{D_{j}\right\}_{j}$ is the set of the double varieties in $\stackrel{\circ}{X}_{i}$. Hence $-K_{X_{i}}-\sum_{j} D_{j}$ is ample. Consequently $-K_{X_{i}}$ is ample.

Remark 3.4. Let $X / \mathbb{F}_{q}$ be a separated scheme of finite type. Assume that $X$ is geometrically connected. By the argument in this section, it is obvious that, if $H^{i}\left(X, \mathcal{O}_{X}\right)=0(\forall i>0)$, then the congruence (1.6.1) holds for $X / \mathbb{F}_{q}$. In particular, if $d=2$, if $X / \mathbb{F}_{q}$ is smooth, if $H^{1}\left(X, \mathcal{O}_{X}\right)=0$ and if $H^{0}\left(X, \Omega_{X / s}^{2}\right)=0$, then the congruence (1.6.1) holds for $X / \mathbb{F}_{q}$. Such an example can be given by a proper smooth Godeaux surface.

Other examples are given by proper smooth unirational threefolds because $H^{i}\left(X, \mathcal{O}_{X}\right)=0(\forall i>0)$ by [53, Introduction, (2.5)].

Let $X / s$ be an SNCL(=simple normal crossing log) classical Enriques surface $X / s$ for $p \neq 2$, i.e., $\left(\Omega_{X / s}^{2}\right)^{\otimes 2}$ is trivial and the corresponding étale covering $X^{\prime}$ to $\Omega_{X / s}^{2}$ is an SNCL K3 surface. (In [46, (7.1)] we have proved that $H^{i}\left(X, \mathcal{O}_{X}\right)=0$ for $i>0$.) Hence the congruence (1.6.1) also holds for $X / s_{\mathbb{F}_{q}}$. See Corollary 5.4 below for the zeta function of this example. By the formulas for the zeta function ((5.4.1), (5.4.2)), we can easily verify that $\# \stackrel{\circ}{X}\left(\mathbb{F}_{q}\right)$ indeed satisfies the congruence (1.6.1). 
More generally, if $H^{i}\left(X, \mathcal{W}\left(\mathcal{O}_{X}\right)\right)_{K_{0}}=0(i>0)$, then the congruence (1.6.1) holds for $X / \mathbb{F}_{q}$ by the proof of Theorem 1.5. By the main theorem of [4], one obtains such examples which are special fibers of regular proper flat schemes over discrete valuation rings of mixed characteristics whose generic fibers are geometrically connected and of Hodge type $\geq 1$ in positive degrees. See also [15] for a generalization of the main theorem in [4].

Example 3.5. Let $n$ and $N$ be positive integers. Set $\mathcal{X}_{1}:=\mathbb{P}_{\mathcal{W}(\kappa)}^{N}$. Blow up $\mathcal{X}_{1}$ along an $\kappa$-rational hyperplane of $\mathbb{P}_{\kappa}^{N}$ and let $\mathcal{X}_{2}$ be the resulting scheme. Let $\stackrel{\circ}{X}_{1}$ and $\stackrel{\circ}{X}_{n}$ be the irreducible components of the special fiber $\mathcal{X}_{2}$. Blow up $\mathcal{X}_{2}$ again along $\stackrel{\circ}{X}_{1} \cap \stackrel{\circ}{X}_{n}$ and let $\mathcal{X}_{3}$ be the resulting scheme. Let $\stackrel{\circ}{X}_{1}, \stackrel{\circ}{X}_{n}$, and $\stackrel{\circ}{X}_{n-1}$ be the irreducible components of the special fiber $\mathcal{X}_{3}$. Blow up $\mathcal{X}_{3}$ again along $\stackrel{\circ}{X}_{1} \cap \stackrel{\circ}{X}_{n-1}$. Continuing this process $(n-1)$-times, we have a projective semistable family $\mathcal{X}_{n}$ over $\operatorname{Spec}(\mathcal{W}(\kappa))$. Let $\stackrel{\circ}{X}_{i}(1 \leq i \leq n)$ be the irreducible components of the special fiber $\mathcal{X}_{n}$. Let $X$ be the $\log$ special fiber of $\mathcal{X}_{n}$. Then $X$ is a projective SNCL scheme over $s$. Let $\stackrel{\circ}{X}^{(i)}(i=0,1)$ be the disjoint union of $(i+1)$-fold intersections of the irreducible components of $\stackrel{\circ}{X}$. Then $\quad \stackrel{\circ}{X}^{(0)}=\mathbb{P}_{\kappa}^{N} \coprod \underbrace{\left(\mathbb{P}_{\kappa}^{N-1} \times_{\kappa} \mathbb{P}_{\kappa}^{1}\right) \coprod \cdots\left(\mathbb{P}_{\kappa}^{N-1} \times_{\kappa} \mathbb{P}_{\kappa}^{1}\right)}_{(n-1) \text {-times }}$ and $\stackrel{\circ}{X}^{(1)}=$ $\underbrace{\mathbb{P}_{\kappa}^{N-1} \coprod \cdots \coprod \mathbb{P}_{\kappa}^{N-1}}_{(n-1) \text {-times }}$. Using the following spectral sequence:

$$
E_{1}^{i j}=H^{j}\left(X^{(i)}, \mathcal{O}_{X^{(i)}}\right) \Rightarrow H^{i+j}\left(X, \mathcal{O}_{X}\right)
$$


and noting that the dual graph of $\stackrel{\circ}{X}$ is a segment, we see that $H^{i}\left(X, \mathcal{O}_{X}\right)=0(i>0)$. If $s=s_{\mathbb{F}_{q}}$, then it is easy to check that

$$
\begin{aligned}
\stackrel{\circ}{X}\left(\mathbb{F}_{q}\right) & =\frac{q^{N+1}-1}{q-1}+(n-1) \frac{q^{N}-1}{q-1} \frac{q^{2}-1}{q-1}-(n-1) \frac{q^{N}-1}{q-1} \\
& =\frac{q^{N+1}-1}{q-1}+(n-1) q \frac{q^{N}-1}{q-1} .
\end{aligned}
$$

In particular, $\# \stackrel{\circ}{X}\left(\mathbb{F}_{q}\right) \equiv 1 \bmod q$.

The restriction of $\omega_{X / s}$ to $\stackrel{\circ}{X}_{i}$ is isomorphic to $\mathcal{O}_{X_{i}}(-N)$ for $i=0, N$ and $\underset{X_{i}}{\mathcal{O}}(-(N-1))$ for $0<i<N$. Hence $\omega_{X / s}^{-1}$ is ample if $N \geq 2$. Since each $\stackrel{\circ}{X}_{i}$ is $F$-split (the $F$-splitting is given by the " $p^{-1}$-th power" of the canonical coordinate of $\stackrel{\circ}{X}_{i}$ (see $\left.[6,(1.1 .5)]\right)$ ) and because we have the following exact sequence:

$$
0 \rightarrow \mathcal{O}_{X} \rightarrow \bigoplus_{i=1}^{N} \mathcal{O}_{X_{i}} \rightarrow \bigoplus_{i=1}^{N-1} \mathcal{O}_{X_{i} \cap X_{i+1}}
$$

$\stackrel{\circ}{X}$ is $F$-split.

\section{Proof of Theorem 1.7}

Let the notations be as in Theorem 1.7. In this section we prove Theorem 1.7. We may assume that $k=1$.

Since $H^{i}\left(Y, \mathcal{O}_{Y}\right)=0$, we see that

$$
H^{i}\left(Y, \mathcal{W}\left(\mathcal{O}_{Y}\right)\right)=\lim _{n} H^{i}\left(Y, \mathcal{W}_{n}\left(\mathcal{O}_{Y}\right)\right)=0
$$


by the same proof as that of Theorem 1.5. Set $\bar{Y}:=Y \otimes_{\mathbb{F}_{q}} \overline{\mathbb{F}}_{q}$ and $e:=\log _{p} q$. By [2, II (4.3)], the Dieudonné module $M:=D\left(\Phi_{Y / \kappa}\right)$ of $\Phi_{Y / \kappa}$ is equal to $H^{d}\left(Y, \mathcal{W}\left(\mathcal{O}_{Y}\right)\right)$. Let $h$ be the height of $\Phi_{Y / \kappa}$. Hence $\Phi_{Y / \kappa}$ is a commutative formal Lie group over $\kappa$ of dimension 1 and the Dieudonné module $M$ is a free $\mathcal{W}$-module of rank $h$ if $h<\infty \quad([25, \mathrm{~V}$ (28.3.10)]). Let $F: M \rightarrow M$ be the operator " $F$ " on the Dieudonne module $M$. By abuse of notation, we denote the induced morphism $M_{K_{0}} \rightarrow M_{K_{0}}$ by $F$. By (2.0.8), we have the following congruence:

$$
\# Y\left(\mathbb{F}_{q}\right) \equiv 1+\operatorname{Tr}\left(F^{e} \mid M_{K_{0}}\right) \quad \bmod q
$$

in $\overline{\mathcal{W}\left(\mathbb{F}_{q}\right)}$. Set $m:=\operatorname{ord}_{p}\left(\operatorname{Tr}\left(F^{e} \mid M_{K_{0}}\right)\right)$. If $m \leq e=\operatorname{ord}_{p}(q)$, then we obtain the following congruence by (4.0.2):

$$
\# Y\left(\mathbb{F}_{q}\right) \equiv 1 \quad \bmod p^{\lceil m\rceil}
$$

in $\mathbb{Z}$.

First we give the proof of Theorem 1.7 (1).

\section{Proof of Theorem 1.7 (1)}

Assume that $h=\infty$. Then $D\left(\Phi \bar{Y}_{\bar{Y}} \overline{\mathbb{F}}_{q}\right)$ is $\mathcal{W}\left(\overline{\mathbb{F}}_{q}\right)$-torsion. By [2, II (4.3)], $H^{d}\left(\bar{Y}, \mathcal{W}\left(\mathcal{O}_{\bar{Y}}\right)\right)_{K_{0}}=D\left(\Phi_{\bar{Y} / \overline{\mathbb{F}}_{q}}\right)_{K_{0}}=0$. By [28, I (1.9.2)], $\mathcal{W}\left(\mathcal{O}_{\bar{Y}}\right)$ $=\mathcal{W}\left(\mathcal{O}_{Y}\right) \otimes_{\mathcal{W}\left(\mathbb{F}_{q}\right)} \mathcal{W}\left(\overline{\mathbb{F}}_{q}\right)$. Since $\stackrel{\circ}{Y}$ is separated, we obtain the following equality $H^{d}\left(Y, \mathcal{W}\left(\mathcal{O}_{\bar{Y}}\right)\right)=H^{d}\left(Y, \mathcal{W}\left(\mathcal{O}_{Y}\right)\right) \otimes_{\mathcal{W}\left(\mathbb{F}_{q}\right)} \mathcal{W}\left(\overline{\mathbb{F}}_{q}\right)$ by using Čech cohomologies. Hence

$$
H^{d}\left(Y, \mathcal{W}\left(\mathcal{O}_{Y}\right)\right)_{K_{0}\left(\mathbb{F}_{q}\right)}=0
$$


(To obtain this vanishing, one may use the fact that the Dieudonné module commutes with base change (cf. the description of $D\left(\Phi_{Y / \mathbb{F}_{q}}\right)$ in [43, p. 309]).) By (2.0.8) this means the congruence (1.7.1).

Now assume that $h<\infty$. Next we give the proof Theorem 1.7 (2).

\section{Proof of Theorem 1.7 (2)}

Let us recall the following well-known observation ([38, Exercise $6.13])$ :

Proposition 4.1. Let $G$ be a commutative formal Lie group of dimension 1 over a perfect field $\kappa$ of characteristic $p>0$. Assume that the height $h$ of $G$ is finite. Then the slopes of the Dieudonne module of $D(G)$ is $1-h^{-1}$.

Proof. Let $D(\kappa)$ be the Cartier-Dieudonné algebra over $\kappa$. We may assume that $\kappa$ is algebraically closed. In this case, the height is the only invariant which determines the isomorphism class of a 1-dimensional commutative formal group law over $\kappa([25,(19.4 .1)])$. Hence $D(G) \simeq$ $D(\kappa) / D(\kappa)\left(F-V^{h-1}\right) \quad\left(\left[67, \quad\right.\right.$ p. 266]). Express $F\left(1, V, \cdots, V^{h-1}\right)=$ $\left(1, V, \cdots, V^{h-1}\right) A$, where $A \in M_{h}(\mathcal{W})$ (as if $F$ were $\mathcal{W}$-linear). Then $\operatorname{det}(t I-A)=t^{h}-p^{h-1}$. Hence the slopes of $D(G)$ is $\operatorname{ord}_{p}\left(\left(p^{h-1}\right)^{h^{-1}}\right)=$ $1-h^{-1}$.

By Proposition 4.1 and (4.0.3), we obtain the following congruence:

$$
\# Y\left(\mathbb{F}_{q}\right) \equiv 1 \quad \bmod p^{\left\lceil e\left(1-h^{-1}\right)\right\rceil}
$$

in $\mathbb{Z}$.

Lastly we give the proof of Theorem 1.7 (3) in the following. 


\section{Proof of Theorem 1.7 (3)}

Let $\kappa$ be a perfect field of characteristic $p>0$. Let $Y$ be a proper scheme over $\kappa$ of pure dimension $d \geq 1$. (We do not assume that $Y$ is smooth over $\kappa$. ) Assume that $H^{d}\left(Y, \mathcal{O}_{Y}\right) \simeq \kappa$ and that $H^{d-1}\left(Y, \mathcal{O}_{Y}\right)=0$ if $d \geq 2$. Then the following morphism:

$$
H^{d}\left(Y, \mathcal{W}\left(\mathcal{O}_{Y}\right)\right) / p \rightarrow H^{d}\left(Y, \mathcal{O}_{Y}\right)
$$

is an isomorphism. Indeed, this is surjective and

$$
\operatorname{dim}_{\kappa}\left(H^{d}\left(Y, \mathcal{W}\left(\mathcal{O}_{Y}\right)\right) / p\right)=\operatorname{dim}_{\kappa}(M / p)=1=\operatorname{dim}_{\kappa} H^{d}\left(Y, \mathcal{O}_{Y}\right) .
$$

Since $\quad h=1, F \quad$ on $\quad H^{d}\left(Y, \mathcal{W}\left(\mathcal{O}_{Y}\right)\right) \otimes_{\mathcal{W}(\kappa)} \mathcal{W}(\bar{\kappa}) \quad$ is an isomorphism.

Hence $\quad F: H^{d}\left(Y, \mathcal{O}_{Y}\right) \rightarrow H^{d}\left(Y, \mathcal{O}_{Y}\right)$ is an isomorphism. Hence $\# Y\left(\mathbb{F}_{q}\right) \equiv 1+\alpha \bmod q$ for a unit $\alpha \in \mathcal{W}\left(\mathbb{F}_{q}\right)^{*}$. Now Theorem 1.7 (3) follows.

Remark 4.2. (1) If $H^{i}\left(Y, \mathcal{O}_{Y}\right)=0$ for $0<i<d-1$ (this is stronger than (b) and (c) in the Introduction), then Theorem 1.7 (3) also follows from Fulton's trace formula ([19]):

$$
\# Y\left(\mathbb{F}_{q}\right) \bmod p \equiv \sum_{i=0}^{d}(-1)^{i} \operatorname{Tr}\left(F_{q}^{*} \mid H^{i}\left(Y, \mathcal{O}_{Y}\right)\right) \in \mathbb{F}_{q}
$$

(cf. [3, Proposition 5.6]).

(2) Let $X / s$ be a log Calabi-Yau scheme. In [55, (10.1)] we have proved a fundamental equality $h^{F}(X / \kappa)=h(X / \kappa)$. Hence $X$ is quasi- $F$ split (resp., $F$-split) if and only if $h(X / \kappa)<\infty$ (resp., $h(X / \kappa)=1$ ).

Though the following corollary immediately follows from [4, (1.6)], we state it for the convenience of our remembrance. 
Corollary 4.3. Let $Y$ be as in Theorem 1.7. Let $f: Z_{1} \rightarrow Z_{2}$ be a morphism of proper schemes over $\mathbb{F}_{q}$. Assume that $Z_{1}$ or $Z_{2}$ is isomorphic to $Y$ over $\mathbb{F}_{q}$. Assume that $\Phi_{Z_{i} / \mathbb{F}_{q}}(i=1,2)$ is representable. If the pull-back $f^{*}: H^{i}\left(Z_{2}, \mathcal{O}_{Z_{2}}\right) \rightarrow H^{i}\left(Z_{1}, \mathcal{O}_{Z_{1}}\right)$ is an isomorphism, then the natural morphism $\Phi_{Z_{2} / \mathbb{F}_{q}} \rightarrow \Phi_{Z_{1} / \mathbb{F}_{q}}$ is an isomorphism. In particular, $h\left(Z_{1} / \mathbb{F}_{q}\right)=h\left(Z_{2} / \mathbb{F}_{q}\right)$ and Theorem 1.7 for $\# Z_{i}\left(\mathbb{F}_{q}\right)$ holds.

Proof. By the assumption, we have an isomorphism $f^{*}: H^{i}$ $\left(Z_{2}, \mathcal{W}\left(\mathcal{O}_{Z_{2}}\right)\right) \stackrel{\sim}{\rightarrow} H^{i}\left(Z_{1}, \mathcal{W}\left(\mathcal{O}_{Z_{1}}\right)\right)$. Hence the natural morphism $D\left(\Phi_{Z_{2} / \mathbb{F}_{q}}\right) \rightarrow D\left(\Phi_{Z_{1} / \mathbb{F}_{q}}\right)$ is an isomorphism. By Cartier theory, the natural morphism $\Phi_{Z_{2} / \mathbb{F}_{q}} \rightarrow \Phi_{Z_{1} / \mathbb{F}_{q}}$ is an isomorphism. This implies that $h\left(Z_{2} / \mathbb{F}_{q}\right)=h\left(Z_{2} / \mathbb{F}_{q}\right)$ and Theorem 1.7 for $\# Z_{i}\left(\mathbb{F}_{q}\right)$ holds.

The following corollary immediately follows from the proof of $[4,(6.12)]$.

Corollary 4.4. Let $Y$ be as in (1.7). Let $G$ be a finite group acting on $Y / \mathbb{F}_{q}$ such that each orbit of $G$ is contained in an affine open subscheme of $Y$. If $\# G$ is prime to $p$ and the induced action on $H^{d}\left(Y, \mathcal{O}_{Y}\right)$ is trivial, then $h\left((Y / G) / \mathbb{F}_{q}\right)=h\left(Y / \mathbb{F}_{q}\right)$ and Theorem 1.7 for $\#(Y / G)\left(\mathbb{F}_{q}\right)$ holds.

Example 4.5. We give examples of the Theorem 1.7 in trivial logarithmic cases.

(1) Let $E / \mathbb{F}_{p}$ be an elliptic curve. It is very well-known that $E / \mathbb{F}_{p}$ is supersingular if and only if $\# E\left(\mathbb{F}_{p}\right)=p+1$ if $p \geq 5$ ([60, V Exercises 5.9]). As observed in [3, Example 5.11], this also follows from the purity of the weight for an elliptic curve over $\mathbb{F}_{p}:\left|\# E\left(\mathbb{F}_{p}\right)-(p+1)\right| \leq 2 \sqrt{p}$ and Fulton's trace formula. In fact, we can say more in Proposition 4.8 below. 
(2) Let $d \geq 3$ be a positive integer such that $d \neq \equiv 0 \bmod p$. Consider a smooth Calabi-Yau variety $\mathcal{X} / \mathcal{W}\left(\mathbb{F}_{q}\right)$ in $\mathbb{P}_{\mathcal{W}\left(\mathbb{F}_{q}\right)}^{d-1}$ defined by the following equation:

$$
a_{0} T_{0}^{d}+\cdots+a_{d-1} T_{d-1}^{d}=0 \quad\left(a_{0}, \ldots, a_{d-1} \in \mathcal{W}\left(\mathbb{F}_{q}\right)^{*}\right) .
$$

Set $a:=a_{0} \ldots a_{d-1} \in \mathcal{W}\left(\mathbb{F}_{q}\right)$. Let $X / \mathbb{F}_{q}$ be the reduction $\bmod p$ of $\mathcal{X} / \mathcal{W}\left(\mathbb{F}_{q}\right)$. By [62, Theorem 1] (see also [loc. cit., Example 4.13]), the logarithm $l(t)$ of $\Phi_{\mathcal{X}} / \mathcal{W}\left(\mathbb{F}_{q}\right)$ is given by the following formula:

$$
l(t)=\sum_{m=0}^{\infty} a^{m} \frac{(m d) !}{(m !) d} \frac{t^{m d+1}}{m d+1} .
$$

(a) If $p \equiv 1 \bmod d$, then

$$
p l(t)=p t+p \sum_{i=2}^{p-1} c_{i} t^{i}+(\text { unit }) t^{p}+\left(\text { higher terms than } t^{p}\right)
$$

for some $c_{i} \in \mathcal{W}\left(\mathbb{F}_{q}\right)$ in $\mathcal{W}\left(\mathbb{F}_{q}\right)[[t]]$. Hence $l^{-1}(p l(t)) \bmod p \equiv t^{p}+\cdots$ and the height of $\Phi_{X / \mathbb{F}_{q}}$ is equal to 1 .

(b) If $p \not \equiv 1 \bmod d$, then $p l(t) \in p \mathcal{W}\left(\mathbb{F}_{q}\right)[[t]]$. Hence the height of $\Phi_{X / \mathbb{F}_{q}}$ is equal to $\infty$.

(a) and (b) above are much easier and much more direct proofs of [68, Theorem 5.1].

(3) Especially consider the case $d=4$ in (2) and let $X / \mathbb{F}_{p}$ be a closed subscheme of $\mathbb{P}_{\mathbb{F}_{p}}^{3}$ defined by the following equation:

$$
T_{0}^{4}+T_{1}^{4}+T_{2}^{4}+T_{3}^{4}=0
$$


(a) If $p=3$, then $\# X\left(\mathbb{F}_{3}\right) \equiv 1 \bmod 3$ by Theorem 1.7 (1). In fact, it is easy to see that $\# X\left(\mathbb{F}_{3}\right)=4=1+3^{2}-3 \times 2$. (This $X$ and $X$ in (c) are Tate's examples in [63] of a supersingular K3-surface (in the sense of Shioda) over $\mathbb{F}_{3}$ and $\mathbb{F}_{7}$ ), respectively.)

(b) If $p=5$, then $\# X\left(\mathbb{F}_{5}\right) \neq \equiv 1 \bmod 5$ by Theorem 1.7 (3). In fact, it is easy to see that $\# X\left(\mathbb{F}_{5}\right)=0$. More generally, for a power $q$ of a prime number $p$, let $X_{q}$ be a closed subscheme of $\mathbb{P}_{\mathbb{F}_{q}}^{q-1}$ defined by the following equation:

$a_{0} T_{0}^{q-1}+\cdots+a_{q-2} T_{q-2}^{q-1}=0\left(a_{0}, \ldots, a_{q-2} \in \mathbb{F}_{q}^{*},\left(a_{0}, \ldots, a_{q-2}\right) \neq(0, \ldots, 0)\right)$, where $a_{0}, \ldots, a_{q-2}$ satisfying the following condition: for any nonempty set $I$ of $\{0, \ldots, q-2\}, \sum_{j \in I} a_{j} \neq 0$ in $\mathbb{F}_{q}$. Then $\# X_{q}\left(\mathbb{F}_{q}\right)=0$.

(c) If $p=7$, then $\# X\left(\mathbb{F}_{7}\right) \equiv 1 \bmod 7$ by Theorem 1.7 (1). In fact, one can check that $\# X\left(\mathbb{F}_{7}\right)=64=1+7^{2}+7 \times 2$. In general, if $\Phi\left(X / \mathbb{F}_{q}\right)$ is supersingular, then $\# X\left(\mathbb{F}_{q}\right)=1+q^{2}+q \alpha$ for some $|\alpha| \leq 22$ by the purity of the weight and by $b_{2}\left(\bar{X} / \bar{F}_{q}\right)=22$. Here $b_{2}\left(\bar{X} / \overline{\mathbb{F}}_{q}\right)$ is the second Betti number of $\bar{X} / \overline{\mathbb{F}}_{q}$. (We do not know an example of the big $|\alpha|$.

(4) See $[70,(4.8)]$ for explicit examples of $X / \mathbb{F}_{q}$ 's such that $h\left(\Phi_{X / \mathbb{F}_{q}}\right)=2$. See also [68, Section 6].

Example 4.6. (1) Let $n$ be a positive integer. Let $X$ be an $n$-gon over $\mathbb{F}_{q}$. Then, by $[50,(6.7)(1)], X$ is $F$-split. In particular, $h^{F}(X)=$ $h\left(X / \mathbb{F}_{q}\right)=1$. Then, by Theorem $1.7(3), \# X\left(\mathbb{F}_{q}\right) \neq \equiv 1 \bmod p$. In fact, it is easy to see that $\# X\left(\mathbb{F}_{q}\right)=n(q+1)-n=n q$. Compare this example with the Example 3.5. 
(2) Let $\kappa$ be a perfect field of characteristic $p>0$. Let $X$ be an SNCL(= simple normal crossing log) K3-surface over $\kappa$, that is, an SNCL Calabi-Yau variety of dimension 2 ([46]). In [50, (6.7) (2)] we have proved the following:

(a) If $\stackrel{\circ}{X}$ is of Type II ([46, Section 3]), then $X$ is $F$-split if and only if the isomorphic double elliptic curve is ordinary. In this case, $h(X / \kappa)=1$. If this is not the case, $h(X / \kappa)=2$.

(b) If $\stackrel{\circ}{X}$ is of Type III ([loc. cit.]), then $X$ is $F$-split and $h(X / \kappa)=1$.

See Corollary 5.2 below for the zeta function of these examples. By the formulas for the zeta functions ((5.2.1) and (5.2.2)), we can easily verify that $\# \stackrel{\circ}{X}\left(\mathbb{F}_{q}\right)$ indeed satisfies the congruences (1.7.3) and (1.7.2).

Remark 4.7. (1) Let $X / \mathbb{F}_{q}$ and $X^{*} / \mathbb{F}_{q}$ be a strong mirror CalabiYau pair in the sense of Wan ([72]), whose strict definition has not been given. Then he conjectures that $\# X\left(\mathbb{F}_{q}\right) \equiv \# X^{*}\left(\mathbb{F}_{q}\right) \bmod q([72,(1.3)])$. Hence the following question seems natural: does the equality $h\left(\Phi_{X / \mathbb{F}_{q}}\right)=h\left(\Phi_{X^{*} / \mathbb{F}_{q}}\right)$ hold? If his conjecture is true, only one of $h\left(\Phi_{X / \mathbb{F}_{q}}\right)$ and $h\left(\Phi_{X^{*} / \mathbb{F}_{q}}\right)$ cannot be 1 by Theorem 1.7. This is compatible with Wan's generically ordinary conjecture in [loc. cit., (8.3)].

(2) If $X$ satisfies the conditions (a), (c) and (d) in the Introduction and if $X$ is a special fiber of a regular proper flat scheme over a discrete valuation ring of mixed characteristics whose generic fibers are geometrically connected and of Hodge type $\geq 1$ in degrees in $[1, d-2]$, then we see that $X$ satisfies the condition (b) in the Introduction by [4].

We conclude this section by generalizing Example (4.5) (1) by using Theorem 1.7 and raise an important question: 
Proposition 4.8. Let $C$ be a proper smooth curve over $\mathbb{F}_{q}$ such that $H^{0}\left(C, \mathcal{O}_{C}\right) \simeq \mathbb{F}_{q} \simeq H^{1}\left(C, \mathcal{O}_{C}\right)$. Recall that $e=\log _{p} q$. Then the following hold:

(1) Assume that $e$ is odd and $p \geq 5$. Then $h_{C / \mathbb{F}_{q}}=2$ if and only if $\# C\left(\mathbb{F}_{q}\right)=1+q$

(2) Assume that $e$ is odd and $p=3$ or 2 . Then $h_{C / \mathbb{F}_{q}}=2$ if and only if $\# C\left(\mathbb{F}_{q}\right)=1+q$ or $1+q \pm p^{\frac{e+1}{2}}$.

(3) Assume that $e$ is even. Then $h_{C / \mathbb{F}_{q}}=2$ if and only if $\# C\left(\mathbb{F}_{q}\right)=$ $1+q+\alpha p^{\frac{e}{2}}$, where $\alpha \in \mathbb{N}$ and $|\alpha| \leq 2$.

Proof. By the purity of weight, we have the following inequality:

$$
\left|\# C\left(\mathbb{F}_{q}\right)-(1+q)\right| \leq 2 \sqrt{q} .
$$

(1) Assume that $h_{C / \mathbb{F}_{q}}=2 . \operatorname{By}(1.7 .2), \# C\left(\mathbb{F}_{q}\right) \equiv 1 \bmod p^{\left\lceil\frac{e}{2}\right\rceil}=1 \bmod p^{\frac{e+1}{2}}$. Hence $\# C\left(\mathbb{F}_{q}\right)=1+m p^{\frac{e+1}{2}}$ for $m \in \mathbb{N}$. By (4.8.1) we have the following inequality:

$$
p^{\frac{1}{2}}\left|m-p^{\frac{e-1}{2}}\right| \leq 2
$$

Since $p \geq 5, m=p^{\frac{e-1}{2}}$. Hence $\# C\left(\mathbb{F}_{q}\right)=1+q$.

Conversely, assume that $\# C\left(\mathbb{F}_{q}\right)=1+q$. Then $C$ can be an elliptic curve over $\mathbb{F}_{q}$. Hence $h_{C / \mathbb{F}_{q}}=1$ or 2 ([60, IV (7.5)]). By (1.7.3) and (1.7.2), $h_{C / \mathbb{F}_{q}}=2$. 
(2) Assume that $h_{C / \mathbb{F}_{q}}=2$. Then, by (4.8.2), $m=p^{\frac{e-1}{2}}$ or $m= \pm 1+$ $p^{\frac{e-1}{2}}$. Hence $\# C\left(\mathbb{F}_{q}\right)=1+q$ or $\# C\left(\mathbb{F}_{q}\right)=1+\left( \pm 1+p^{\frac{e-1}{2}}\right) p^{\frac{e+1}{2}}=1+q$ $\pm p^{\frac{e+1}{2}}$.

The proof of the converse implication is the same as that in (1).

(3) Assume that $h_{C / \mathbb{F}_{q}}=2$. By (1.7.2), \#C( $\left.\mathbb{F}_{q}\right) \equiv 1 \bmod p^{\frac{e}{2}}$. Hence $\# C\left(\mathbb{F}_{q}\right)=1+m p^{\frac{e}{2}}$ for $m \in \mathbb{N}$. By (4.8.1),

$$
\left|m-p^{\frac{e}{2}}\right| \leq 2
$$

Hence, by (4.8.1), $m=\alpha+p^{\frac{e}{2}}$ with $|\alpha| \leq 2$. Hence $\# C\left(\mathbb{F}_{q}\right)=1+(\alpha+$ $\left.p^{\frac{e}{2}}\right) p^{\frac{e}{2}}=1+q+\alpha p^{\frac{e}{2}}$.

The proof of the converse implication is the same as that in (1).

Remark 4.9. Assume that $e$ is even. By Honda-Tate's theorem for elliptic curves over finite fields (Theorem 4.10 below), the case $|\alpha|=1$ occurs only when $p \not \equiv 1 \bmod 3$; the case $\alpha=0$ occurs only when $p \not \equiv 1 \bmod 4$.

Theorem 4.10 (Honda-Tate's theorem for elliptic curves ([73, (4.1)], $[56,(4.8)]))$. For an elliptic curve $E / \mathbb{F}_{q}$, set $t_{E}:=1+q-\# E\left(\mathbb{F}_{q}\right)$. Consider the following well-defined injective map: $\left\{\right.$ isogeny classes of elliptic curves $\left.E / \mathbb{F}_{q}\right\} \ni E \rightarrow t_{E} \in\{t \in \mathbb{Z}|| t \mid \leq 2 \sqrt{q}\}$.

(This map is indeed injective by Tate's theorem ([64, Main Theorem]).) The image of this map consists of the following values:

(1) $t$ is coprime to $p$. 
(2) $e$ is even and $t= \pm 2 \sqrt{q}$.

(3) $e$ is even and $p \neq 1 \bmod 3$ and $t= \pm \sqrt{q}$.

(4) $e$ is odd and $p=2$ or 3 and $t= \pm p^{\frac{e+1}{2}}$.

(5) $e$ is odd, or $e$ is even and $p \neq \equiv 1 \bmod 4$ and $t=0$.

The case (1) arises from ordinary elliptic curves over $\mathbb{F}_{q}$. The case (2) arises from supersingular elliptic curves over $\mathbb{F}_{q}$ having all their endomorphisms defined over $\mathbb{F}_{q}$; the rest cases arises from supersingular elliptic curves over $\mathbb{F}_{q}$ not having all their endomorphisms defined over $\mathbb{F}_{q}$.

Problem 4.11. Let $K$ be an algebraic number field and $\mathcal{O}_{K}$ the integer ring of $K$. Let $x$ be a positive real number.

(1) Consider the following set:

$\mathcal{P}(x):=\left\{\mathfrak{p} \in \operatorname{Spec}\left(\mathcal{O}_{K}\right) \mid N_{K / \mathbb{Q}}(\mathfrak{p}) \leq x\right.$ and $\log _{p}\left(\#\left(\mathcal{O}_{K} / \mathfrak{p}\right)\right)$ is even $\}$, where $p=\operatorname{ch}\left(\mathcal{O}_{K} / \mathfrak{p}\right)$.

Assume that $p \geq 5$. Let $E / K$ be an elliptic curve. Let $\alpha$ be an integer such that $|\alpha| \leq 2$. Consider the following set:

$\mathcal{P}^{\prime}(x ; E / K, \alpha):=\left\{\mathfrak{p} \in \mathcal{P}(x) \mid E\right.$ has a good reduction $\mathcal{E}_{0}$ at $\mathfrak{p}$ and $\left.\# \mathcal{E}_{0}\left(\mathbb{F}_{q}\right)=1+q+\alpha \sqrt{q}\right\}$.

Set

$$
\mathcal{P}(x ; E / K, \alpha):= \begin{cases}\mathcal{P}^{\prime}(x ; E / K, \alpha) & (|\alpha|=2), \\ \{\mathfrak{p} \in \mathcal{P}(x ; E / K, \alpha) \mid p \neq \equiv 1 \bmod 3\} & (|\alpha|=1), \\ \{\mathfrak{p} \in \mathcal{P}(x ; E / K, \alpha) \mid p \neq \equiv 1 \bmod 4\} & (\alpha=0) .\end{cases}
$$


Then, what is the function

$$
x \mapsto \frac{\# \mathcal{P}(x ; E / K, \alpha)}{\# \mathcal{P}(x)}
$$

when $x \rightarrow \infty$ ? (I do not know whether $\lim _{x \rightarrow \infty} \mathcal{P}(x ; E / K, \alpha)=\infty$ for each $\alpha$ such that $|\alpha| \leq 2$ for any non-CM elliptic curve over $K$ (see [61, p. 185, Exercise 2.33 (a), (b)] for a CM elliptic curve over $\mathbb{Q}(\sqrt{-1})$ : in this example, $\lim _{x \rightarrow \infty} \mathcal{P}(x ; E / K, 2)=\infty$, but $\mathcal{P}(x ; E / K, \alpha)=0$ for $\alpha \neq 2$ and for any $x$ ). If $[K: \mathbb{Q}]$ is odd or if $K$ has a real embedding, then $\lim _{x \rightarrow \infty} \sum_{|\alpha| \leq 2} \mathcal{P}(x ; E / K, \alpha)=\infty$ by Elkies' theorems ([13, Theorem 2], [14, Theorem]).)

When $p=2$ or 3 , we can give a similar problem to the problem above by using Proposition 4.8 (2).

(2) Consider the following set:

$$
\mathcal{P}(x):=\left\{\mathfrak{p} \in \operatorname{Spec}\left(\mathcal{O}_{K}\right) \mid N_{K / \mathbb{Q}}(\mathfrak{p}) \leq x\right\} .
$$

Let $S / K$ be a K3 surface. Let $\alpha$ be an integer such that $|\alpha| \leq 22$. Consider the following set:

$$
\begin{array}{r}
\mathcal{P}^{\prime}(x ; S / K, \alpha):=\left\{\mathfrak{p} \in \mathcal{P}(x) \mid S \text { has a good reduction } \mathcal{S}_{0} \text { at } \mathfrak{p}\right. \\
\text { and } \left.\# \mathcal{S}_{0}\left(\mathbb{F}_{q}\right)=1+q^{2}+\alpha q\right\} .
\end{array}
$$

Then, what is the function

$$
x \mapsto \frac{\# \mathcal{P}^{\prime}(x ; S / K, \alpha)}{\# \mathcal{P}(x)}
$$

when $x \rightarrow \infty$ ? (I do not know even whether $\lim _{x \rightarrow \infty} \sum_{|\alpha| \leq 22} \mathcal{P}^{\prime}(x$; $S / K, \alpha)=\infty$. 


\section{Two Kinds of Zeta Functions of Degenerate SNCL Schemes over the Log Point of $\mathbb{F}_{q}$}

In this section, we give a few examples of two kinds of local zeta functions of a separated scheme $Y$ of finite type over $\mathbb{F}_{q}$ : one of them is defined by rational points of $Y$; the other is defined by the Kummer étale cohomology of $Y$ when $Y$ is the underlying scheme of a proper log smooth scheme over the log point $s_{\mathbb{F}_{q}}$.

First we introduce a Grothendieck group which is convenient in this section.

Let $F$ be a field. Consider a Grothendieck group $\mathcal{K}(F)$ with the following generators and relations: the generators of $\mathcal{K}(F)$ are $[(V, \beta)]$ 's, where $V$ is a finite-dimensional vector space over $F$ and $\beta$ is an endomorphism of $V$ over $F$. The relations are as follows: $[(V, \beta)]=$ $[(U, \alpha)]+[(W, \gamma)]$ for a commutative diagram with exact rows

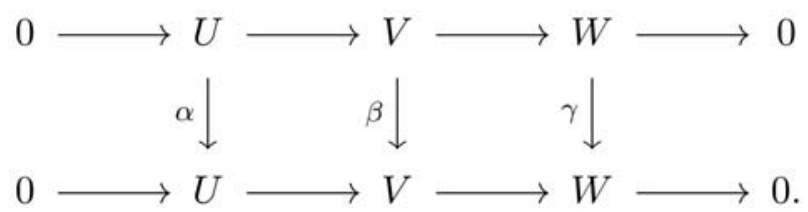

Let $t$ be a variable. Note that $\operatorname{det}(1-t \beta \mid V)=\operatorname{det}(1-t \alpha \mid U) \operatorname{det}(1-t \gamma \mid W)$. If $V=\{0\}$, we set $\operatorname{det}(1-t 0 \mid V)=1 \quad(1 \in F)$. We have a natural map

$$
\operatorname{det}(1-t \bullet \mid \bullet): \mathcal{K}(F) \rightarrow F(t)^{*} \cap(1+t F[[t]])^{*}
$$

of abelian groups. Here the intersection in the target of (5.0.1) is considered in the ring of Laurent power series in one variable with coefficients in $F$. Set $Z((V, \alpha), t)=\operatorname{det}(1-t \alpha \mid V)$.

Let $Y$ be a separated scheme of finite type over $\mathbb{F}_{q}$. Set

$$
\left[\left(E_{p}\left(Y / \mathbb{F}_{q}\right), F_{q}^{*}\right)\right]:=\sum_{i=0}^{\infty}(-1)^{i}\left[\left(H_{\mathrm{rig}, c}^{i}\left(Y / K_{0}\left(\mathbb{F}_{q}\right)\right), F_{q}^{*}\right)\right] \in \mathcal{K}\left(K_{0}\left(\mathbb{F}_{q}\right)\right)
$$


where $E_{p}$ means the Euler-characteristic. Let

$$
Z\left(Y / \mathbb{F}_{q}, t\right):=\exp \left(\sum_{n=0}^{\infty} \frac{\# Y\left(\mathbb{F}_{q^{n}}\right)}{n} t^{n}\right)
$$

be the zeta function of $Y / \mathbb{F}_{q}$. We can reformulate (2.0.1) as the following formula:

$$
Z\left(Y / \mathbb{F}_{q}, t\right)=Z\left(\left[\left(E_{p}\left(Y / \mathbb{F}_{q}\right), F_{q}^{*}\right)\right]\right)^{-1} .
$$

Proposition 5.1. Let $Y$ be a proper SNC (not necessarily log) scheme over $\mathbb{F}_{q}$. Let $Y^{(i)}\left(i \in \mathbb{Z}_{\geq 0}\right)$ be the disjoint union of the $(i+1)$-fold intersections of the irreducible components of $Y$. Then

$$
Z\left(Y / \mathbb{F}_{q}, t\right)=\prod_{i, j \geq 0} \operatorname{det}\left(1-t F_{q}^{*} \mid H_{\text {rig }}^{j}\left(Y^{(i)} / K_{0}\left(\mathbb{F}_{q}\right)\right)\right)^{(-1)^{i+j+1}} .
$$

Proof. Let $Y$. be the Čech diagram of an affine open covering of $Y$ by finitely many affine open subschemes $U_{j}$ 's of $Y$. Let $\stackrel{\circ}{Y}_{1}, \ldots, \stackrel{\circ}{Y}_{N}$ be the irreducible components of $\stackrel{\circ}{Y}$. Set $U_{j}^{(i)}:=\coprod_{0 \leq k_{0}<k_{1}<\cdots<k_{l} \leq N}$ $\left(\stackrel{\circ}{Y}_{k_{0}} \cap \ldots \cap \stackrel{\circ}{Y}_{k_{l}} \cap U_{j}\right), Y_{0}^{(i)}:=\coprod_{j} U_{j}^{(i)}$ and $Y_{n}^{(i)}:=\operatorname{cosk}_{0}^{Y^{(i)}}\left(Y_{0}^{(i)}\right)_{n}(n \in \mathbb{N})$. Let $Y_{\bullet} \stackrel{\subseteq}{\rightarrow} \mathcal{P}$. be a closed immersion into a formally smooth formal scheme over $\operatorname{Spf}\left(\mathcal{W}\left(\mathbb{F}_{q}\right)\right)$. Then we have a closed immersion $Y_{0}^{(i)} \stackrel{\subseteq}{\rightarrow} \coprod^{(i)} \mathcal{P}_{0}$, where $\coprod^{(i)} \mathcal{P}_{0}$ is a finite sum of $\mathcal{P}_{0}$ which depends on $i$. Let $\delta_{j}: Y_{0}^{(i+1)} \rightarrow Y_{0}^{(i)}(0 \leq j \leq i)$ be the standard face morphism. Then we have a natural morphism $\Delta_{j}: \coprod^{(i+1)} \mathcal{P}_{0} \rightarrow \coprod^{(i)} \mathcal{P}_{0}$ fitting into the following commutative diagram: 


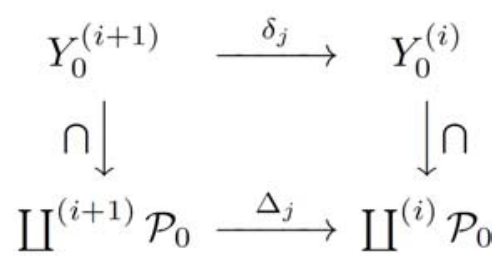

and satisfying the standard relations. Set $\mathcal{P}_{\bullet}^{(i)}:=\operatorname{cosk}_{0}^{\mathcal{W}\left(\mathbb{F}_{q}\right)}\left(\coprod^{(i)} \mathcal{P}_{0}\right)$. Let sp: $] Y_{\bullet}^{(i)}\left[\mathcal{P}_{\bullet}^{(i)} \rightarrow Y_{\bullet}^{(i)}\right.$ be the specialization map. Then, as in [8, (2.3)], the following sequence:

$$
0 \rightarrow \operatorname{sp}_{*}\left(\Omega_{Y_{\bullet}\left[\mathcal{P}_{\bullet}\right.}^{\bullet}\right) \rightarrow \operatorname{sp}_{*}\left(\Omega_{j Y_{\bullet}^{(0)}\left[{ }_{\mathcal{P}}^{(0)}\right.}\right) \rightarrow \operatorname{sp}_{*}\left(\Omega_{j Y_{\bullet}^{(1)}\left[\mathcal{P}_{\bullet}^{(1)}\right.}\right) \rightarrow \cdots
$$

is exact. Hence we have the following spectral sequence:

$$
E_{1}^{i j}=H_{\text {rig }}^{j}\left(Y^{(i)} / K_{0}\left(\mathbb{F}_{q}\right)\right) \Rightarrow H_{\text {rig }}^{i+j}\left(Y / K_{0}\left(\mathbb{F}_{q}\right)\right)
$$

By (5.0.4) and this spectral sequence, we obtain the following formula:

$$
\left.\left[\left(E_{p}\left(Y / \mathbb{F}_{q}\right), F_{q}^{*}\right)\right]=\sum_{i, j \geq 0}(-1)^{i+j}\left[H_{\text {rig }}^{j}\left(Y^{(i)} / K_{0}\left(\mathbb{F}_{q}\right)\right), F_{q}^{*}\right)\right] \in \mathcal{K}\left(K_{0}\left(\mathbb{F}_{q}\right)\right) .
$$

This formula implies (5.1.1).

Corollary 5.2. Let $X / \mathbb{F}_{q}$ be a non-smooth combinatorial K3 surface ([22, 36, 46]). (We do not assume that X has a log structure of simple normal crossing type.) Let $m$ be the summation of the times of the processes of blowing downs making all irreducible components relatively minimal. Let $M_{1}$ (resp., $M_{2}$ ) be the cardinality of the irreducible components of $\stackrel{\circ}{X}$ whose relatively minimal models are $\mathbb{P}_{\mathbb{F}_{q}}^{2}$ (resp., Hirzeburch surfaces $=$ relatively minimal rational ruled surfaces). Let $M$ be the cardinality of the irreducible components of $\stackrel{\circ}{X}$. Then the following hold: 
(1) If $X$ is of Type II with double elliptic curve $E / \mathbb{F}_{q}$, then $Z\left(\stackrel{\circ}{X} / \mathbb{F}_{q}, t\right)=\frac{\operatorname{det}\left(1-q t F_{q}^{*} \mid H_{\text {rig }}^{1}\left(E / K_{0}\left(\mathbb{F}_{q}\right)\right)\right)^{M-2}}{(1-t) \operatorname{det}\left(1-t F_{q}^{*} \mid H_{\text {rig }}^{1}\left(E / K_{0}\left(\mathbb{F}_{q}\right)\right)\right)(1-q t)^{M_{1}+2 M_{2}+M-3+m}\left(1-q^{2} t\right)^{M}}$.

(2) Assume that $X$ is of Type III. Let $d$ be the cardinality of the double curves of $\stackrel{\circ}{X}$. Then

$$
Z\left(\stackrel{\circ}{X} / \mathbb{F}_{q}, t\right)=\frac{1}{(1-t)^{2}(1-q t)^{M_{1}+2 M_{2}+m-d}\left(1-q^{2} t\right)^{M}} .
$$

Proof. First we give a remark on the rigid cohomology of a smooth projective rational surface $S$ over $\mathbb{F}_{q}$. Set $S_{\mathbb{F}_{q^{n}}}:=S \underset{\mathbb{F}_{q}}{\otimes \mathbb{F}_{q^{n}}}$ and $S_{\overline{\mathbb{F}}_{q}}:=$ $S \underset{\mathbb{F}_{q}}{\otimes} \overline{\mathbb{F}}_{q}$

Let $\bar{S}_{\text {min }}$ be a relatively minimal model of $S_{\overline{\mathbb{F}}_{q}}$. If $\bar{S}_{\min } \simeq \mathbb{P}_{\bar{F}_{q}}^{2}$, we see that the motive $H\left(S_{\overline{\mathbb{F}}_{q}}\right)$ is as follows by $([12,(6.12)]$ :

$$
H\left(S_{\overline{\mathbb{F}}_{q}}\right) \simeq H\left(\mathbb{P}_{\mathbb{F}_{q}}^{2}\right) \oplus H(D)(-1),
$$

where $D$ is the disjoint sum of 0 -dimensional points. Since $H^{2}\left(\mathbb{P}_{\mathbb{F}_{q}}^{2}\right)$ is isomorphic to a Tate-twist, if a natural number $n$ is big enough, then $\left(F_{q}^{*}\right)^{n}$ on $H_{\text {rig }}^{2}\left(S_{\mathbb{F}_{q^{n}}} / K_{0}\left(\mathbb{F}_{q^{n}}\right)\right)$ is $\operatorname{diag}\left(q^{n}, \cdots, q^{n}\right)$. Hence the eigenvalues of $\left(F_{q}^{*}\right)^{n}$ are $q^{n}(n \gg 0)$ and thus the eigenvalues of $F_{q}^{*}$ are $q$.

If $\bar{S}_{\text {min }}$ is isomorphic to a relatively minimal ruled surface over a smooth curve $C$ over $\overline{\mathbb{F}}_{q}$, the motive $H\left(S_{\overline{\mathbb{F}}_{q}}\right)$ is as follows by $([12,(6.10)$, (6.12)]:

$$
H\left(S_{\overline{\mathbb{F}}_{q}}\right) \simeq H(C) \oplus H(C)(-1) \oplus H(D)(-1)
$$

Hence we see that $F_{q}^{*}$ on $H_{\text {rig }}^{2}\left(S / K_{0}\left(\mathbb{F}_{q}\right)\right)$ is $\operatorname{diag}(q, \ldots, q)$ as above. 
(1) It is easy to check that

$$
H_{\text {rig }}^{i}\left(\stackrel{\circ}{X^{(0)}} / K_{0}\left(\mathbb{F}_{q}\right)\right)= \begin{cases}K_{0}\left(\mathbb{F}_{q}\right)^{M} & (i=0), \\ H_{\text {rig }}^{1}\left(E / K_{0}\left(\mathbb{F}_{q}\right)\right)^{\oplus M-2} & (i=1), \\ K_{0}\left(\mathbb{F}_{q}\right)(-1)^{M_{1}+2 M_{2}+2(M-2)+m} & (i=2), \\ H_{\text {rig }}^{1}\left(E / K_{0}\left(\mathbb{F}_{q}\right)\right)(-1)^{\oplus M-2} & (i=3), \\ K_{0}\left(\mathbb{F}_{q}\right)(-2)^{M} & (i=4),\end{cases}
$$

and

$$
H_{\text {rig }}^{i}\left(\stackrel{\circ}{X}(1) / K_{0}\left(\mathbb{F}_{q}\right)\right)= \begin{cases}K_{0}\left(\mathbb{F}_{q}\right)^{M-1} & (i=0), \\ H_{\text {rig }}^{1}\left(E / K_{0}\left(\mathbb{F}_{q}\right)\right)^{\oplus M-1} & (i=1), \\ K_{0}\left(\mathbb{F}_{q}\right)(-1)^{M-1} & (i=2) .\end{cases}
$$

Now (5.2.1) follows from (5.1.1).

(2) Let $T$ be the cardinality of the triple points of $\stackrel{\circ}{X}$. It is easy to check that

$$
\begin{aligned}
& H_{\text {rig }}^{i}\left(\stackrel{\circ}{X}^{(0)} / K_{0}\left(\mathbb{F}_{q}\right)\right)= \begin{cases}K_{0}\left(\mathbb{F}_{q}\right)^{M} & (i=0), \\
0 & (i=1), \\
K_{0}\left(\mathbb{F}_{q}\right)(-1)^{M_{1}+2 M_{2}+m} & (i=2), \\
0 & (i=3), \\
K_{0}\left(\mathbb{F}_{q}\right)(-2)^{M} & (i=4),\end{cases} \\
& H_{\text {rig }}^{i}\left(\stackrel{\circ}{X}^{(1)} / K_{0}\left(\mathbb{F}_{q}\right)\right)= \begin{cases}K_{0}\left(\mathbb{F}_{q}\right)^{d} & (i=0), \\
0 & (i=1), \\
K_{0}\left(\mathbb{F}_{q}\right)(-1)^{d} & (i=2),\end{cases}
\end{aligned}
$$


and

$$
H_{\text {rig }}^{0}\left(\stackrel{\circ}{X}^{(2)} / K_{0}\left(\mathbb{F}_{q}\right)\right)=K_{0}\left(\mathbb{F}_{q}\right)^{T}
$$

Because the dual graph of $\stackrel{\circ}{X}$ is a circle, $M-d+T=\chi\left(\mathbb{S}^{1}\right)=2$. Now (5.2.2) follows from (5.1.1).

Remark 5.3. If $p \neq 2$, we can prove that $T$ is even (cf. [22]). However we do not use this fact in this article.

Corollary 5.4. Assume that $p \neq 2$. Let $X / \mathbb{F}_{q}$ be a non-smooth combinatorial classical Enriques surface ([36, 46]). Let $M_{1}, M_{2}, M, m$ and $d$ be as in (5.2). Then the following hold:

(1) If $X$ is of Type II with double elliptic curve $E / \mathbb{F}_{q}$, then

$$
Z\left(\stackrel{\circ}{X} / \mathbb{F}_{q}, t\right)=\frac{\operatorname{det}\left(1-q t F_{q}^{*} \mid H_{r i g}^{1}\left(E / K_{0}\left(\mathbb{F}_{q}\right)\right)\right)^{M-1}}{(1-t)(1-q t)^{M_{1}+2 M_{2}+M-1+m}\left(1-q^{2} t\right)^{M}}
$$

(2) Assume that $X$ is of Type III. Let $d$ and $T$ be the cardinalities of the double curves of $\stackrel{\circ}{X}$ and the triple points of $\stackrel{\circ}{X}$, respectively. Then

$$
Z\left(\stackrel{\circ}{X} / \mathbb{F}_{q}, t\right)=\frac{1}{(1-t)(1-q t)^{M_{1}+2 M_{2}+m-d}\left(1-q^{2} t\right)^{M}}
$$

Proof. (1) It is easy to check that

$$
H_{\text {rig }}^{i}\left(\stackrel{\circ}{X}^{(0)} / K_{0}\left(\mathbb{F}_{q}\right)\right)= \begin{cases}K_{0}\left(\mathbb{F}_{q}\right)^{M} & (i=0), \\ H_{\text {rig }}^{1}\left(E / K_{0}\left(\mathbb{F}_{q}\right)\right)^{\oplus M-1} & (i=1), \\ K_{0}\left(\mathbb{F}_{q}\right)(-1)^{M_{1}+2 M_{2}+2(M-1)+m} & (i=2), \\ H_{\text {rig }}^{1}\left(E / K_{0}\left(\mathbb{F}_{q}\right)\right)(-1)^{\oplus M-1} & (i=3), \\ K_{0}\left(\mathbb{F}_{q}\right)(-2)^{M} & (i=4),\end{cases}
$$


and

$$
H_{\text {rig }}^{i}\left(\stackrel{\circ}{X}(1) / K_{0}\left(\mathbb{F}_{q}\right)\right)= \begin{cases}K_{0}\left(\mathbb{F}_{q}\right)^{M-1} & (i=0), \\ H_{\text {rig }}^{1}\left(E / K_{0}\left(\mathbb{F}_{q}\right)^{\oplus M-1}\right. & (i=1), \\ K_{0}\left(\mathbb{F}_{q}\right)(-1)^{M-1} & (i=2) .\end{cases}
$$

(2) It is easy to check that

$$
\begin{aligned}
& H_{\text {rig }}^{i}\left(\stackrel{\circ}{X^{(0)}} / K_{0}\left(\mathbb{F}_{q}\right)\right)= \begin{cases}K_{0}\left(\mathbb{F}_{q}\right)^{M} & (i=0), \\
0 & (i=1), \\
K_{0}\left(\mathbb{F}_{q}\right)(-1)^{M_{1}+2 M_{2}+m} & (i=2), \\
0 & (i=3), \\
K_{0}\left(\mathbb{F}_{q}\right)(-2)^{M} & (i=4),\end{cases} \\
& H_{\text {rig }}^{i}\left(\stackrel{\circ}{X}{ }^{(1)} / K_{0}\left(\mathbb{F}_{q}\right)\right)= \begin{cases}K_{0}\left(\mathbb{F}_{q}\right)^{d} & (i=0), \\
0 & (i=1), \\
K_{0}\left(\mathbb{F}_{q}\right)(-1)^{d} & (i=2),\end{cases}
\end{aligned}
$$

and

$$
H_{\text {rig }}^{0}\left(\stackrel{\circ}{X^{(2)}} / K_{0}\left(\mathbb{F}_{q}\right)\right)=K_{0}\left(\mathbb{F}_{q}\right)^{T}
$$

Because the dual graph of $\stackrel{\circ}{X}$ is $\mathbb{P}^{2}(\mathbb{R}), M-d+T=\chi\left(\mathbb{P}^{2}(\mathbb{R})\right)=1$.

Lastly we consider another type of local zeta functions.

Let $\mathcal{V}$ be a complete discrete valuation ring of mixed characteristics with finite residue field $\mathbb{F}_{q}$ and let $K$ be the fraction field of $\mathcal{V}$. Let $\mathfrak{Y}$ be a proper smooth scheme over $K$ of dimension $d$ and let $I$ be the inertia 
group of the absolute Galois group $\operatorname{Gal}(\bar{K} / K)$. Then the zeta function of $\mathfrak{Y}$ is defined as follows:

$$
Z(\mathfrak{Y}, t):=\prod_{i=0}^{2 d} \operatorname{det}\left(1-t \sigma \mid H_{\mathrm{et}}^{i}\left(\mathfrak{Y} \underset{K}{\otimes} \bar{K}, \mathbb{Q}_{l}\right)^{I}\right)^{(-1)^{h+1}}
$$

where $\sigma \in \operatorname{Gal}(\bar{K} / K)$ is a lift of the geometric Frobenius of $\operatorname{Gal}\left(\overline{\mathbb{F}}_{q} / \mathbb{F}_{q}\right)$ and $l$ is a prime which is prime to $q$. If $\mathfrak{Y}$ is the generic fiber of a proper semistable family $\mathcal{Y}$ over $\mathcal{V}$ with special fiber $Y$, then the following formula holds by [23] ([29]):

$$
Z(\mathfrak{Y}, t)=\prod_{h=0}^{2 d} \operatorname{det}\left(1-t \sigma \mid H_{\mathrm{ket}}^{h}\left(\bar{Y}, \mathbb{Q}_{l}\right)^{I}\right)^{(-1)^{i+1}} .
$$

Let $\mathcal{X}$ be a proper strict semistable family of surfaces over $\mathcal{V}$ with $\log$ special fiber $X$ over $s_{\mathbb{F}_{q}}$.

Then [41, (6.3.3)] tells us that $Z\left(\mathcal{X}_{K}, t\right)$ can be described by the log crystalline cohomologies by the coincidence of the monodromy filtration and the weight filtration. ([48, (8.3)], [41, (6.2.4)]; however see $[47,(11.15)]$ and $[48,(7.1)]$.

$$
Z\left(\mathcal{X}_{K}, t\right)=\prod_{i=0}^{4} \operatorname{det}\left(1-t F_{q}^{*} \mid\left(H_{\text {crys }}^{i}\left(X / \mathcal{W}\left(s_{\mathbb{F}_{q}}\right)\right)_{K_{0}\left(\mathbb{F}_{q}\right)}\right)^{N=0}\right)^{(-1)^{i+1}}
$$

where $\mathcal{W}\left(s_{\mathbb{F}_{q}}\right)$ is the canonical lift of $s_{\mathbb{F}_{q}}$ over $\mathcal{W}\left(\mathbb{F}_{q}\right), H_{\text {crys }}^{i}(X / \mathcal{W}$ $\left.\left(s_{\mathbb{F}_{q}}\right)\right)$ is the $i$-th log crystalline cohomology of $X / \mathcal{W}\left(\mathbb{F}_{q}\right)$ and

$$
N: H_{\text {crys }}^{i}\left(X / \mathcal{W}\left(s_{\mathbb{F}_{q}}\right)\right)_{K_{0}\left(\mathbb{F}_{q}\right)} \rightarrow H_{\text {crys }}^{i}\left(X / \mathcal{W}\left(s_{\mathbb{F}_{q}}\right)\right)_{K_{0}\left(\mathbb{F}_{q}\right)}(-1)
$$

is the $p$-adic monodromy operator. More generally, for a proper SNCL scheme $Y / s_{\mathbb{F}_{q}}$ of pure dimension $d$, set

$$
Z\left(H^{i}\left(Y / K_{0}\left(s_{\mathbb{F}_{q}}\right)\right), t\right):=\operatorname{det}\left(1-t F_{q}^{*} \mid\left(H_{\mathrm{crys}}^{i}\left(X / \mathcal{W}\left(s_{\mathbb{F}_{q}}\right)\right)_{K_{0}\left(\mathbb{F}_{q}\right)}\right)^{N=0}\right)^{(-1)^{i+1}}
$$


and

$$
Z\left(Y / s_{\mathbb{F}_{q}}, t\right):=\prod_{i=0}^{2 d} Z\left(H^{i}\left(Y / K_{0}\left(s_{\mathbb{F}_{q}}\right)\right), t\right)^{(-1)^{i+1}}
$$

Let us recall the following result due to the author ([52, (8.3)], (cf. [40, (2.2)], [9, (6.4)])):

Theorem 5.5 ([52, (8.3)]). Let $\kappa$ be a perfect field of characteristic $p>0$. Let $X / s$ be an SNCL K3 surface. Let $H_{\log }^{i}(X)(i \in \mathbb{N})$ be the $i$-th log crystalline cohomology or the i-th Kummer étale cohomology of X/s. Then the following hold:

(1) The $\star$-adic $(\star=p, l)$ monodromy filtration and the weight one on $H_{\log }^{i}(X)$ coincide.

(2) The following hold:

(a) X is of Type I if and only if $N=0$ on $H_{\log }^{2}(X)$.

(b) $X$ is of Type II if and only if $N \neq 0$ and $N^{2}=0$ on $H_{\log }^{2}(X)$.

(c) $X$ is of Type III if and only if $N^{2} \neq 0$ on $H_{\log }^{2}(X)$.

Proof. For the completeness of this article, we give the proof of Theorem 5.5.

We give the proof of this theorem in the $p$-adic case because the proof in the $l$-adic case is the same as that in the $p$-adic case.

Recall the following weight spectral sequence ([41, 3.23], [47, (2.0.1)]):

$$
E_{1}^{-k, i+k}=\bigoplus_{j \geq \max \{-k, 0\}} H_{\text {rig }}^{i-2 j-k}\left(\stackrel{\circ}{X}^{(2 j+k)} / K_{0}\right)(-j-k) \Rightarrow H_{\text {crys }}^{i}(X / \mathcal{W}(s))_{K_{0}} .
$$


(See [47] for the mistakes in [41].) Here we have used Berthelot's comparison isomorphism $H_{\text {crys }}^{i}(Y / \mathcal{W})_{K_{0}}=H_{\text {rig }}^{i}\left(Y / K_{0}\right)(i \in \mathbb{N})$ for a proper smooth scheme $Y$ over $\kappa$. By [47, (3.6)] this spectral sequence degenerates at $E_{2}$. (The $l$-adic analogue of this spectral sequence also degenerates at $E_{2}$ by Nakayama's theorem ([45, (2.1)]).)

(1) We may assume that $\kappa$ is algebraically closed. If $X / s$ is of Type I, there is nothing to prove.

If $X$ is of Type III, the double curves and the irreducible components are rational, and hence $E_{1}^{0,1}=E_{1}^{1,1}=E_{1}^{0,3}=E_{1}^{-1,3}=0$. By [46, (3.5) (3)], $H_{\log -\text { crys }}^{1}(X / \mathcal{W})=0$ and hence we have $E_{2}^{-1,2}=0$. (Note that we also have the similar vanishing for the first Kummer étale cohomology of $X$ by the vanishing above and the existence of the $\mathbb{Q}$-structure of $E_{2}^{-1,2}$ (cf. the proof of [48, (8.3)])). By taking the duality in [47, (10.5)], $E_{2}^{1,2}=0$. By $[41,6.2 .1]$, the $p$-adic monodromy operator $N: H_{\text {crys }}^{2}(X / \mathcal{W}(s)) \rightarrow H_{\text {crys }}^{2}(X / \mathcal{W}(s))(-1)$ induces an isomorphism $N^{2}: E_{2}^{-2,4} \stackrel{\sim}{\rightarrow} E_{2}^{2,0}(-2)=K_{0}$

If $X$ is of Type II, then $E_{1}^{-2,4}=E_{1}^{2,0}=0$. By [46, (3.5) (3)] again, $H_{\log -\text { crys }}^{1}(X / \mathcal{W})=H_{\log -\text { crys }}^{3}(X / \mathcal{W})=0$. Hence $E_{2}^{i j}=0$ for $i+j=1,3$. Because $N: H_{\text {crys }}^{2}(X / \mathcal{W}(s)) \rightarrow H_{\text {crys }}^{2}(X / \mathcal{W}(s))(-1)$ induces an isomorphism $E_{2}^{-1,3} \stackrel{\cong}{\rightarrow} E_{2}^{1,1}(-1)$ by [41, 6.2.2], we have proved (1).

(2) (2) follows from (1) and the non-vanishings of $E_{2}^{1,1}$ in the Type II case and $E_{2}^{2,0}$ in the Type III case, respectively. 
Remark 5.6. The author has found the Theorem 5.5 in December 1996 by using the $p$-adic weight spectral sequence (5.5.1). The key point of the proof is to notice to use the $p$-adic weight spectral sequence of $X / s$ instead of the Clemens-Schmid exact sequence used in Kulikov's article [36]. (In fact, the complex analogue (5.7) below of (5.5) holds; this is a generalization of Kulikov's theorem in [loc. cit.] and the proof of (5.5) is simpler than that in [loc. cit.]. To my surprise, mathematicians who are working over $\mathbb{C}$ have not used the weight spectral sequence (5.7.1).) The author has finished writing the preprint [52] by 2000 at the latest (cf. [45, Remark 2.4 (3)]). However, after that, he has noticed that there are too many non-minor mistakes in theory of log de Rham-Witt complexes in Hyodo-Kato's article [26] and Mokrane's article [41] as pointed out in [47]. Because he has used Hyodo-Kato's and Mokrane's theory in [52] heavily, he has to use their results in correct ways. However he has used his too much time for correcting their results in [47], he has no will to publish [52] now (because [52] is quite long and because he has to use more time for adding comments about Hyodo-Kato's and Mokrane's articles in [52]). For example, $v$ in [41] is not a morphism of complexes, the left $N$ in the diagram in [40, (2.2)] is incorrect.

In [40, (2.2)]. Matsumoto has proved (5.5) for semistable algebraic spaces of K3-surfaces after looking at the proof in [52]. (See "Proof of $p$-adic case" in the proof of [40, Proposition 2.2].)

Theorem 5.7 (cf. [36]). Let $s$ be the log point of $\mathbb{C}$. Let $X / s$ be an analytic SNCL K3 surface. Let $X_{\infty}$ be the base change of the KatoNakayama space $X^{\log }$ of $X$ ([37]) with respect to the morphism $\mathbb{R} \ni x$ $\mapsto \exp (2 \pi \sqrt{-1}) \in \mathbb{S}^{1}$. Let $N: H^{i}\left(X_{\infty}, \mathbb{Q}\right) \rightarrow H^{i}\left(X_{\infty}, \mathbb{Q}\right)(-1)(i \in \mathbb{N})$ be the monodromy operator constructed in [21]. Then the following hold:

(1) The weight filtration on $H^{i}\left(X_{\infty}, \mathbb{Q}\right)$ constructed in [21] coincide with the monodromy filtration on $H^{i}\left(X_{\infty}, \mathbb{Q}\right)$ 
(2) The following hold:

(a) $X$ is of Type I if and only if $N=0$ on $H^{2}\left(X_{\infty}, \mathbb{Q}\right)$.

(b) $X$ is of Type II if and only if $N \neq 0$ and $N^{2}=0$ on $H^{2}\left(X_{\infty}, \mathbb{Q}\right)$.

(c) $X$ is of Type III if and only if $N^{2} \neq 0$ on $H^{2}\left(X_{\infty}, \mathbb{Q}\right)$.

Proof. By $[48,(2.1 .10)]$ we have the following weight spectral sequence:

$$
E_{1, \infty}^{-k, h+k}=\underset{j \geq \max \{-k, 0\}}{\bigoplus} H^{h-2 j-k}\left(\stackrel{\circ}{X}^{(2 j+k+1)}, \mathbb{Q}\right)(-j-k) \Rightarrow H^{h}\left(X_{\infty}, \mathbb{Q}\right) .
$$

By [18, (5.9)], if $X$ is a combinatorial Type II or Type III K3 surface over $\mathbb{C}$, then $H^{0}\left(X, \Omega_{X / \mathbb{C}}^{1}\right)=0$. (Of course, if $X$ is of Type $\mathrm{I}$, then $H^{0}\left(X, \Omega_{X / \mathbb{C}}^{1}\right)=0$ by Hodge symmetry.) Hence $H^{1}\left(X_{\infty}, \mathbb{C}\right)=H_{\mathrm{dR}}^{1}$ $(X / \mathbb{C})=H^{0}\left(X, \Omega_{X / s}^{1}\right) \oplus H^{1}\left(X, \mathcal{O}_{X}\right)=0$. Here we have used the isomorphism between Steenbrink compleces $A_{\mathbb{Q}} \otimes_{\mathbb{Q}} \mathbb{C}$ and $A_{\mathbb{C}}$ of $X$ and the isomorphism between $A_{\mathbb{C}}$ and $\Omega_{X / s}^{\bullet}$ ([21]). By the duality of the $E_{2}$-terms of (5.7.1) ([48, (5.15) (2)]) and the degeneration at $E_{2}$ of (5.7.1) (by Hodge theory), we obtain the vanishing of $H^{3}\left(X_{\infty}, \mathbb{C}\right)$. The rest of the proof is the same as that of (5.5).

Theorem 5.8 ([52, (15.1)]). Let $X / s_{\mathbb{F}_{q}}$ be a projective SNCL K3 surface. Then the following hold:

(1)

$$
Z\left(H^{i}\left(X / K_{0}\left(s_{\mathbb{F}_{q}}\right)\right), t\right)= \begin{cases}1-t & (i=0), \\ 1 & (i=1,3), \\ 1-q^{2} t & (i=4) .\end{cases}
$$


(2) If $X$ is of Type II with double elliptic curve $E$, then

$$
Z\left(H^{2}\left(X / K_{0}\left(s_{\mathbb{F}_{q}}\right)\right), t\right)=\operatorname{det}\left(1-t F_{q}^{*} \mid H_{\text {rig }}^{1}\left(E / K_{0}\right)\right)(1-q t)^{18} .
$$

Consequently,

$$
Z\left(X / s_{\mathbb{F}_{q}}, t\right)=\frac{1}{(1-t) \operatorname{det}\left(1-t F_{q}^{*} \mid H_{\text {rig }}^{1}\left(E / K_{0}\right)\right)(1-q t)^{18}\left(1-q^{2} t\right)} .
$$

(3) If $X$ is of Type III, then

$$
Z\left(H^{2}\left(X / K_{0}\left(s_{\mathbb{F}_{q}}\right)\right), t\right)=(1-t)(1-q t)^{19} .
$$

Consequently

$$
Z\left(X / s_{\mathbb{F}_{q}}, t\right)=\frac{1}{(1-t)^{2}(1-q t)^{19}\left(1-q^{2} t\right)} .
$$

Proof. (1) By $[46,(3.5)], \quad H_{\text {crys }}^{i}\left(X / \mathcal{W}\left(s_{\mathbb{F}_{q}}\right)\right)=0(i=1,3)$. Thus $Z\left(H^{i}\left(X / K_{0}\left(s_{\mathbb{F}_{q}}\right)\right), t\right)=1(i=1,3)$. By $[46, \quad(6.9)], \quad X$ is the log special fiber of a projective semistable family $\mathcal{X}$ over $\operatorname{Spec}\left(W\left(\mathbb{F}_{q}\right)\right)$. By [46, (6.10)], the generic fiber of $\mathcal{X}$ is a K3 surface. Hence, by Hyodo-Kato's isomorphism ([26, (5.1)] (however see [47, Section 7] for incompleteness of the proof of Hyodo-Kato isomorphism), $\operatorname{dim}_{K_{0}\left(\mathbb{F}_{q}\right)} H_{\text {crys }}^{2}\left(X / \mathcal{W}\left(s_{\mathbb{F}_{q}}\right)\right)_{K_{0}\left(\mathbb{F}_{q}\right)}=22$.

(2) In this case, by (5.5), $N \neq 0, N: E_{2}^{-1,2} \rightarrow E_{2}^{1,1}$ is an isomorphism, $N^{2}=0$ on $H_{\text {crys }}^{2}\left(X / \mathcal{W}\left(s_{\mathbb{F}_{q}}\right)\right)_{K_{0}\left(\mathbb{F}_{q}\right)}$ and $E_{2}^{-2,4}=E_{2}^{2,0}=0$. Hence we have the following exact sequence by (5.5):

$$
0 \rightarrow E_{2}^{1,1} \rightarrow \operatorname{Ker}(N) \rightarrow E_{2}^{0,2} \rightarrow 0 .
$$


Because $E_{2}^{1,1} \simeq H_{\text {rig }}^{1}\left(E / K_{0}\left(\mathbb{F}_{q}\right)\right), \operatorname{det}\left(1-t F_{q}^{*} \mid E_{2}^{1,1}\right)=\operatorname{det}\left(1-t F_{q}^{*} \mid H_{\text {rig }}^{1}(E /\right.$ $\left.K_{0}\left(\mathbb{F}_{q}\right)\right)$. On the other hand, $E_{2}^{0,2}$ is a subquotient of

$$
H_{\text {rig }}^{0}\left(X^{(2)} / K_{0}\left(\mathbb{F}_{q}\right)\right)(-1) \oplus H_{\text {rig }}^{2}\left(X^{(1)} / K_{0}\left(\mathbb{F}_{q}\right)\right) .
$$

Hence $F_{q}^{*}$ on $E_{2}^{0,2}$ is $\operatorname{diag}(q, \ldots, q)$ as shown in the proof of (5.2). Since $\operatorname{Ker}(N)$ is 20-dimensional, we obtain (2).

(3) In this case, by (5.5), $N^{2} \neq 0, N^{3}=0$ and $E_{2}^{-1,3}=E_{2}^{1,1}=0$. Because $N^{2}: E_{2}^{-2,4} \rightarrow E_{2}^{2,0}(-2)$ is an isomorphism, $N: E_{2}^{0,2} \rightarrow E_{2}^{2,0}(-1)$ is surjective and hence the kernel of $N$ is 20-dimensional. Obviously $F_{q}^{*}=$ id on $E_{2}^{2,0}$. As in $(2), F_{q}^{*}$ on $E_{2}^{0,2}$ is $\operatorname{diag}(q, \ldots, q)$. Hence we obtain (3).

Theorem 5.9 ([52, (15.2)]). Let $X / s_{\mathbb{F}_{q}}$ be a projective non-smooth SNCL classical Enriques surface. Then

$$
Z\left(H^{i}\left(X / K_{0}\left(s_{\mathbb{F}_{q}}\right)\right), t\right)= \begin{cases}1-t & (i=0), \\ 1 & (i=1,3), \\ (1-q t)^{10} & (i=2), \\ 1-q^{2} t & (i=4) .\end{cases}
$$

Consequently

$$
Z\left(X / s_{\mathbb{F}_{q}}, t\right)=\frac{1}{(1-t)(1-q t)^{10}\left(1-q^{2} t\right)}
$$

Proof. By [46, (7.1)], $H_{\text {crys }}^{i}\left(X / \mathcal{W}\left(s_{\mathbb{F}_{q}}\right)\right)=0(i=1,3)$ and hence $Z\left(H^{i}\left(X / K_{0}\left(s_{\mathbb{F}_{q}}\right)\right), t\right)=1(i=1,3)$. By [46, (7.1)] and the argument in [46, (6.8), (6.11)], $X$ is the $\log$ special fiber of a projective semistable family $\mathcal{X}$ over $\mathcal{W}\left(\mathbb{F}_{q}\right)$ and the generic fiber of $\mathcal{X}$ is a classical Enriques 
surface. Hence $\operatorname{dim}_{K_{0}\left(\mathbb{F}_{q}\right)} H_{\text {crys }}^{2}\left(X / \mathcal{W}\left(s_{\mathbb{F}_{q}}\right)\right)_{K_{0}\left(\mathbb{F}_{q}\right)}=10$. The rest of the proof is the same as that of (5.8) by noting that $0=E_{2}^{-1,3}=$ $E_{2}^{1,1}=E_{2}^{20}=E_{2}^{-2,4}$, where $E_{2}^{\bullet \bullet \bullet}$ s are $E_{2}$-terms of the spectral sequence (5.5.1).

\section{Appendix}

\section{A Remark on Katsura and Van der Geer's Result}

In this section we generalize the argument in the proof of (1.7) (3).

First we recall the following theorem in [55]. This is a generalization of Katsura and Van der Geer's theorem ([67, (5.1), (5.2), (16.4)]).

Theorem 6.1 ([55, (2.3)]). Let $\kappa$ be a perfect field of characteristic $p>0$. Let $Y$ be a proper scheme over $\kappa$. (We do not assume that $Y$ is smooth over $\kappa$.$) Let q$ be a nonnegative integer. Assume that $H^{q}\left(Y, \mathcal{O}_{Y}\right)$ $\simeq \kappa$, that $H^{q+1}\left(Y, \mathcal{O}_{Y}\right)=0$ and that $\Phi_{Y / \kappa}^{q}$ is pro-representable. Assume also that the Bockstein operator

$$
\beta: H^{q-1}\left(Y, \mathcal{O}_{Y}\right) \rightarrow H^{q}\left(Y, \mathcal{W}_{n-1}\left(\mathcal{O}_{Y}\right)\right)
$$

arising from the following exact sequence:

$$
0 \rightarrow \mathcal{W}_{n-1}\left(\mathcal{O}_{Y}\right) \stackrel{V}{\rightarrow} \mathcal{W}_{n}\left(\mathcal{O}_{Y}\right) \rightarrow \mathcal{O}_{Y} \rightarrow 0
$$

is zero for any $n \in \mathbb{Z}_{\geq 2}$. Let $V: \mathcal{W}_{n-1}\left(\mathcal{O}_{Y}\right) \rightarrow \mathcal{W}_{n}\left(\mathcal{O}_{Y}\right)$ be the Verschiebung morphism and let $F: \mathcal{W}_{n}\left(\mathcal{O}_{Y}\right) \rightarrow \mathcal{W}_{n}\left(\mathcal{O}_{Y}\right)$ be the induced morphism by the Frobenius endomorphism of $\mathcal{W}_{n}(Y)$. Let $n^{q}(Y)$ be the minimum of positive integers n's such that the induced morphism

$$
F: H^{q}\left(Y, \mathcal{W}_{n}\left(\mathcal{O}_{Y}\right)\right) \rightarrow H^{q}\left(Y, \mathcal{W}_{n}\left(\mathcal{O}_{Y}\right)\right)
$$


by the $F: \mathcal{W}_{n}\left(\mathcal{O}_{Y}\right) \rightarrow \mathcal{W}_{n}\left(\mathcal{O}_{Y}\right)$ is not zero. (If $F=0$ for all $n$, then set $n^{q}(Y):=\infty$. ) Let $h^{q}(Y / \kappa)$ be the height of the Artin-Mazur formal group $\Phi_{Y / \kappa}^{q}$ of $Y / \kappa$. Then $h^{q}(Y / \kappa)=n^{q}(Y)$.

Proposition 6.2. Let the notations be as in (6.1). Let $D(\kappa)$ be the Cartier-Dieudonné algebra over $\kappa$. Then the following hold:

(1) length ${ }_{\mathcal{W}} H^{q}\left(Y, \mathcal{W}_{n}\left(\mathcal{O}_{Y}\right)\right)=n\left(n \in \mathbb{Z}_{\geq 1}\right)$.

(2) Set $h:=h\left(\Phi_{Y / \kappa}^{q}\right)$. Assume that $h<\infty$. Let us consider the following natural surjective morphism $H^{q}\left(Y, \mathcal{W}\left(\mathcal{O}_{Y}\right)\right) \rightarrow H^{q}\left(Y, \mathcal{W}_{h}\left(\mathcal{O}_{Y}\right)\right)$. Then this morphism induces the following isomorphism:

$$
H^{q}\left(Y, \mathcal{W}\left(\mathcal{O}_{Y}\right)\right) / p \stackrel{\sim}{\rightarrow} H^{q}\left(Y, \mathcal{W}_{h}\left(\mathcal{O}_{Y}\right)\right)
$$

of $D(\kappa) / p$-modules.

Proof. (1) By the assumptions we have the following exact sequence:

$$
0 \rightarrow H^{q}\left(Y, \mathcal{W}_{n-1}\left(\mathcal{O}_{Y}\right)\right) \stackrel{V}{\rightarrow} H^{q}\left(Y, \mathcal{W}_{n}\left(\mathcal{O}_{Y}\right)\right) \rightarrow H^{q}\left(Y, \mathcal{O}_{Y}\right) \rightarrow 0
$$

(1) immediately follows from this.

(2) First assume that $h=1$. Then $H^{q}\left(Y, \mathcal{W}\left(\mathcal{O}_{Y}\right)\right) \simeq \mathcal{W}$. In this case, (2) is obvious.

Next assume that $1<h<\infty$. Set $M_{n}:=H^{q}\left(X, \mathcal{W}_{n}\left(\mathcal{O}_{X}\right)\right)\left(n \in \mathbb{Z}_{\geq 1}\right)$ and $M:=H^{q}\left(X, \mathcal{W}\left(\mathcal{O}_{X}\right)\right)$. Consider the following exact sequence:

$$
0 \rightarrow \mathcal{W}_{m-n}\left(\mathcal{O}_{Y}\right) \stackrel{V^{n}}{\rightarrow} \mathcal{W}_{m}\left(\mathcal{O}_{Y}\right) \rightarrow \mathcal{W}_{n}\left(\mathcal{O}_{Y}\right) \rightarrow 0
$$

for $m>n$. By the assumption and (3.2.1) we see that $H^{q+1}\left(Y, \mathcal{W}_{m}\left(\mathcal{O}_{X}\right)\right)=0$ for any $m$. Hence the natural morphism $M_{m} \rightarrow M_{n}$ is surjective and 
consequently the natural morphism $M \rightarrow M_{n}$ is surjective. In particular, the natural morphism $M \rightarrow M_{h}$ is surjective. Let $\eta$ be an element of $M_{h}$. We claim that $p \eta=0$.

We have to distinguish the operator $F: M_{n} \rightarrow M_{n}$ and the operator $F: M_{n} \rightarrow M_{n-1}$. The latter $F$ is equal to $R_{n} F$, where $R_{n}$ : $M_{n} \rightarrow M_{n-1}$ is the projection. We denote $R_{n} F$ by $F_{n}$ to distinguish two $F$ 's. Since the following diagram

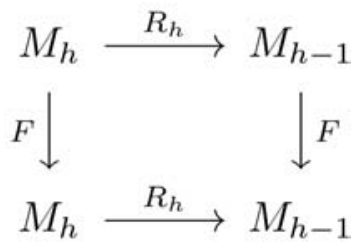

is commutative, we have the following:

$$
p \eta=V F_{h}(\eta)=V R_{h} F(\eta)=V F R_{h}(\eta) .
$$

Since $F=0$ on $M_{h-1}$ by (6.1), the last term is equal to zero. Hence $p \eta=0$. Consequently, the natural morphism $H^{q}\left(Y, \mathcal{W}\left(\mathcal{O}_{Y}\right)\right) \rightarrow H^{q}(Y$, $\left.\mathcal{W}_{h}\left(\mathcal{O}_{Y}\right)\right)$ factors through the projection $H^{q}\left(Y, \mathcal{W}\left(\mathcal{O}_{Y}\right)\right) \rightarrow H^{q}(Y, \mathcal{W}$ $\left.\left(\mathcal{O}_{Y}\right)\right) / p$. Since the morphism (6.2.1) is surjective and $\operatorname{dim}_{\kappa} H^{q}(Y, \mathcal{W}$ $\left.\left(\mathcal{O}_{Y}\right)\right) / p=h=\operatorname{dim}_{\kappa} H^{q}\left(Y, \mathcal{W}_{h}\left(\mathcal{O}_{Y}\right)\right)$ by (1), the morphism (6.2.1) is an isomorphism.

Remark 6.3. Let the notations be as in (1.7) (2). By using only (6.2) for the case $q=d$, we can prove that

$$
\# Y\left(\mathbb{F}_{q^{k}}\right) \equiv 1 \bmod p^{\left[\frac{k e+1}{2}\right]} \quad\left(k \in \mathbb{Z}_{\geq 1}\right),
$$

where [ ] is the Gauss symbol. However the congruence (6.3.1) is not sharper than (1.7.2); only in the case $h=2,(6.3 .1)$ is equivalent to (1.7.2). 


\section{Acknowledgement}

I have begun this work after listening to Nakamaura's very clear talk in which the main theorem in [54] has been explained in the conference "Higher dimensional algebraic geometry" of Kawamata in March 2018 at Tokyo University. The talk of Gongyo in January 2017 at Tokyo Denki University for the explanation of the main theorem in [24] has given a very good influence to this article. Without their talks, I have not begun this work. I would like to express sincere gratitude to them. I would also like to express sincere thanks to Tanaka and Ejiri (resp., Esnault) for their kindness for informing me of the articles [27, 39, 65] (resp., [7]) and giving me an important remark.

\section{References}

[1] J. Ax, Zeroes of polynomials over finite fields, American Journal of Mathematics 86(2) (1964), 255-261.

DOI: https://doi.org/10.2307/2373163

[2] M. Artin and B. Mazur, Formal groups arising from algebraic varieties, Annales Scientifiques de l'École Normale Supérieure, 4e Série 10(1) (1977), 87-131.

DOI: https://doi.org/10.24033/asens.1322

[3] T-H. Bülles, Fulton's trace formula for coherent cohomology, Preprint, Available from.

$$
\text { http://www.math.uni-bonn.de/people/huybrech/Buelles.pdf }
$$

[4] P. Berthelot, S. Bloch and H. Esnault, On Witt vector cohomology for singular varieties, Compositio Mathematica 143(2) (2007), 363-392.

$$
\text { DOI: https://doi.org/10.1112/S0010437X06002533 }
$$

[5] P. Berthelot, H. Esnault and K. Rülling, Rational points over finite fields for regular models of algebraic varieties of Hodge type $\geq 1$, Annals of Mathematics 176(1) (2012), 413-508.

DOI: https://doi.org/10.4007/annals.2012.176.1.8

[6] M. Brion and S. Kumar, Frobenius Splitting Methods in Geometry and Representation Theory, Progress in Mathematics, Birkhäuser, 2005.

[7] A. Chatzistamatiou, On the Frobenius stable part of Witt vector cohomology, Mathematische Annalen 354(3) (2012), 1177-1200.

DOI: https://doi.org/10.1007/s00208-011-0769-6 
[8] B. Chiarellotto, Rigid cohomology and invariant cycles for a semistable log scheme, Duke Mathematical Journal 97(1) (1999), 155-169.

DOI: https://doi.org/10.1215/S0012-7094-99-09707-7

[9] B. Chiarellotto and C. Lazda, Combinatorial degenerations of surfaces and CalabiYau threefolds, Algebra and Number Theory 10(10) (2016), 2235-2266.

DOI: https://doi.org/10.2140/ant.2016.10.2235

[10] B. Chiarellotto and B. Le Stum, Pentes en cohomologie rigide et F-isocristaux unipotents, Manuscripta Mathematica 100(4) (1999), 455-468.

DOI: https://doi.org/10.1007/s002290050212

[11] P. Deligne, La conjecture de Weil: II, Publications Mathématiques de l'Institut des Hautes Études Scientifiques 52(1) (1980), 137-252.

DOI: https://doi.org/10.1007/BF02684780

[12] P. Deligne and J. S. Milne, Tannakian Categories, In: Hodge Cycles, Motives, and Shimura Varieties, Lecture Notes in Mathematics, 900, Springer-Verlag, $2^{\text {nd }}$ Corrected Printing (1989), 101-228.

[13] N. D. Elkies, The existence of infinitely many supersingular primes for every elliptic curve over $\mathbb{Q}$, Inventiones Mathematicae 89(3) (1987), 561-567.

DOI: https://doi.org/10.1007/BF01388985

[14] N. D. Elkies, Supersingular primes for elliptic curves over real number fields, Compositio Mathematica 72(2) (1989), 165-172.

[15] V. Ertl, A new proof of a vanishing result due to Berthelot, Esnault, and Rülling, Appear in Journal of Number Theory (2019).

DOI: https://doi.org/10.1016/j.jnt.2019.03.022

[16] H. Esnault, Varieties over a finite field with trivial Chow group of o-cycles have a rational point, Inventiones Mathematicae 151(1) (2003), 187-191.

DOI: https://doi.org/10.1007/s00222-002-0261-8

[17] J.-Y. Étesse and B. Le Stum, Fonctions $L$ associées aux $F$-isocristaux surconvergents: I, Interprétation cohomologique, Mathematische Annalen 296(1) (1993), 557-576.

DOI: https://doi.org/10.1007/BF01445120

[18] R. Friedman, Global smoothings of varieties with normal crossings, Annals of Mathematics 118(1) (1983), 75-114.

DOI: https://doi.org/10.2307/2006955

[19] W. Fulton, A fixed point formula for varieties over finite fields, Mathematica Scandinavica 42 (1978), 189-196.

DOI: https://doi.org/10.7146/math.scand.a-11747 
[20] E. Freitag and R. Kiehl, Etale Cohomology and the Weil Conjecture, Ergebnisse der Mathematik und Ihrer Grenzgebiete, 3, Springer-Verlag, 1988.

DOI: https://doi.org/10.1007/978-3-662-02541-3

[21] T. Fujisawa and C. Nakayama, Mixed Hodge structures on log deformations, Rendiconti del Seminario Matematico della Università di Padova 110 (2003), 221-268.

[22] R. Friedman and F. Scattone, Type III degenerations of K3 surfaces, Inventiones Mathematicae 83(1) (1986), 1-39.

DOI: https://doi.org/10.1007/BF01388751

[23] K. Fujiwara and K. Kato, Logarithmic etale topology theory, Preprint (1995).

[24] Y. Gongyo, Y. Nakamura and H. Tanaka, Rational points on log Fano threefolds over a finite field, Preprint, Available from arXiv:1512.05003v3.

[25] M. Hazewinkel, Formal Groups and Applications, Pure and Applied Mathematics Series, Academic Press, New York, 1978.

[26] O. Hyodo and K. Kato, Semi-stable reduction and crystalline cohomology with logarithmic poles, In: Périodes p-Adiques, Seminaire de Bures, 1988, Astérisque 223, Soc. Math. de France (1994), 221-268.

[27] W. Haboush and N. Lauritzen, Varieties of unseparated flags, linear algebraic groups and their representations (Los Angeles, 1992), Amer. Math. Soc. (1993), 35-57.

[28] L. Illusie, Complexe de de Rham-Witt et cohomologie cristalline, Annales scientifiques de l'École Normale Supérieure, 4e Série 12(4) (1979), 501-661.

$$
\text { DOI: https://doi.org/10.24033/asens.1374 }
$$

[29] L. Illusie, An overview of the work of K. Fujiwara, K. Kato and C. Nakayama on logarithmic étale cohomology, Societe Mathematique de France, Asterisque 279 (2002), 271-322.

[30] K. Joshi, Exotic torsion, Frobenius splitting and the slope spectral sequence, Canadian Mathematical Bulletin 50(4) (2007), 567-578.

DOI: https://doi.org/10.4153/CMB-2007-054-9

[31] K. Kato, Logarithmic structures of Fontaine-Illusie, In: Algebraic Analysis, Geometry, and Number Theory, Johns Hopkins University Press (1989), 191-224.

[32] K. Kato, Toric singularities, American Journal of Mathematics 116(5) (1994), 1073-1099.

DOI: https://doi.org/10.2307/2374941

[33] N. M. Katz, On a theorem of Ax, American Journal of Mathematics 93(2) (1971), 485-499.

DOI: https://doi.org/10.2307/2373389

[34] M. Kim, A vanishing theorem for Fano varieties in positive characteristic, Preprint, Available from arXiv:math/0201183v3. 
[35] K. Kodaira, On a differential-geometric method in the theory of analytic stacks, Proceedings of the National Academy of Sciences of the United States of America 39(12) (1953), 1268-1273.

DOI: https://doi.org/10.1073/pnas.39.12.1268

[36] V. S. Kulikov, Degenerations of K3 surfaces and Enriques' surfaces, Mathematics of the USSR-Izvestiya 11(5) (1977), 957-989.

DOI: https://doi.org/10.1070/IM1977v011n05ABEH001753

[37] K. Kato and C. Nakayama, Log Betti cohomology, log étale cohomology, and log de Rham cohomology of log schemes over $\mathbb{C}$, Kodai Mathematical Journal 22(2) (1999), 161-186.

DOI: https://doi.org/10.2996/kmj/1138044041

[38] C. Liedtke, Lectures on supersingular K3 surfaces and the crystalline Torelli theorem, In K3 Surfaces and their Moduli, Progress in Mathematics 315 (2016), 171-235.

DOI: https://doi.org/10.1007/978-3-319-29959-4_8

[39] N. Lauritzen and A. P. Rao, Elementary counterexamples to Kodaira vanishing in prime characteristic, Proceedings of the Indian Academy of Sciences - Mathematical Sciences 107(1) (1997), 21-25.

DOI: https://doi.org/10.1007/BF02840470

[40] Y. Matsumoto, Good reduction criterion for K3 surfaces, Mathematische Zeitschrift 279(1-2) (2015), 241-266.

DOI: https://doi.org/10.1007/s00209-014-1365-8

[41] A. Mokrane, La suite spectrale des poids en cohomologie de Hyodo-Kato, Duke Mathematical Journal 72(2) (1993), 301-337.

DOI: https://doi.org/10.1215/S0012-7094-93-07211-0

[42] D. Mumford, Pathologies of modular algebraic surfaces, American Journal of Mathematics 83(2) (1961), 339-342.

DOI: https://doi.org/10.2307/2372959

[43] D. Mumford, Bi-extensions of formal groups, In: Bombay Colloquium on Algebraic Geometry, Oxford University Press (1969), 307-322.

[44] V. B. Mehta and A. Ramanathan, Frobenius splitting and cohomology vanishing for Schubert varieties, Annals of Mathematics 122(1) (1985), 27-40.

DOI: https://doi.org/10.2307/1971368

[45] C. Nakayama, Degeneration of $l$-adic weight spectral sequences, American Journal of Mathematics 122(4) (2000), 721-733.

DOI: https://doi.org/10.1353/ajm.2000.0030

[46] Y. Nakkajima, Liftings of log K3 surfaces and classical log Enriques surfaces in mixed characteristics, Journal of Algebraic Geometry 9 (2000), 355-393. 
[47] Y. Nakkajima, $p$-adic weight spectral sequences of log varieties, Journal of Mathematical Sciences, The University of Tokyo 12(4) (2005), 513-661.

[48] Y. Nakkajima, Signs in weight spectral sequences, monodromy-weight conjectures, log Hodge symmetry and degenerations of surfaces, Rendiconti del Seminario Matematico della Università di Padova 116 (2006), 71-185.

[49] Y. Nakkajima, Weight filtration and slope filtration on the rigid cohomology of a variety in characteristic $p>0$, Mémoires de la Société Mathématique de France 130-131 (2012), pp 256.

DOI: https://doi.org/10.24033/msmf.441

[50] Y. Nakkajima, Artin-Mazur heights and Yobuko heights of proper log smooth schemes of Cartier type, and Hodge-Witt decompositions and Chow groups of quasi$F$-split threefolds, Preprint, Available from arXiv:1902.00185.

[51] Y. Nakkajima, Limits of weight filtrations and limits of slope filtrations on infinitesimal cohomologies in mixed characteristics I, Preprint, Available from arXiv:1902.00182.

[52] Y. Nakkajima, Monodromies and weight filtrations, and simple normal crossing log surfaces with torsion canonical sheaves, Preprint.

[53] N. Nygaard, On the fundamental group of a unirational 3-fold, Inventiones Mathematicae 44(1) (1978), 75-86.

DOI: https://doi.org/10.1007/BF01389903

[54] Y. Nakamura and H. Tanaka, A Witt Nadel vanishing theorem for threefolds, Preprint, Available from arXiv:1712.07358v1.

[55] Y. Nakkajima and F. Yobuko, Degenerations of log Hodge de Rham spectral sequences, log Kodaira vanishing theorem in characteristic $p>0$ and log weak Lefschetz conjecture for log crystalline cohomologies, Preprint, Available from arXiv:1902.09110v2.

[56] M. Papikian, Honda-Tate theorem for elliptic curves, Preprint, Available from.

http://www.math.psu.edu/papikian/Research/HTS.pdf

[57] M. Raynaud, Contre-exemple au "vanishing theorem" en caracteristique $p>0$, C. P. Ramanujam- A Tribute, Tata Institute of Fundamental Research Studies in Mathematics, Springer-Verlag (1978), 273-278.

[58] M. Schlessinger, Functors of Artin rings, Transactions of the American Mathematical Society 130(2) (1968), 208-222.

DOI: https://doi.org/10.1090/S0002-9947-1968-0217093-3

[59] N. I. Shepherd-Barron, Fano threefolds in positive characteristic, Compositio Mathematica 105(3) (1997), 237-265.

DOI: https://doi.org/10.1023/A:1000158618674 
[60] J. H. Silverman, The Arithmetic of Elliptic Curves (2nd Edition), Graduate Texts in Mathematics 106, Springer-Verlag, 2009.

[61] J. H. Silverman, Advanced Topics in the Arithmetic of Elliptic Curves, Graduate Texts in Mathematics 151, Springer-Verlag, 1994.

[62] J. Stienstra, Formal group laws arising from algebraic varieties, American Journal of Mathematics 109(5) (1987), 907-925.

DOI: https://doi.org/10.2307/2374494

[63] J. Tate, Algebraic cycles and poles of zeta functions, In: Arithmetic Algebraic Geometry, Harper and Row, New York (1965), 93-110.

[64] J. Tate, Endomorphisms of abelian varieties over finite fields, Inventiones Mathematicae 2(2) (1966), 134-144.

DOI: https://doi.org/10.1007/BF01404549

[65] B. Totaro, The failure of Kodaira vanishing for Fano varieties, and terminal singularities that are not Cohen-Macaulay, Preprint, Available from arXiv:1710.04364v1.

[66] T. Tsuji, Poincaré duality for logarithmic crystalline cohomology, Compositio Mathematica 118(1) (1999), 11-41.

DOI: https://doi.org/10.1023/A:1001020809306

[67] G. Van der Geer and T. Katsura, On a stratification of the moduli of K3 surfaces, Journal of the European Mathematical Society 2(3) (2000), 259-290.

DOI: https://doi.org/10.1007/s100970000021

[68] G. Van der Geer and T. Katsura, On the height of Calabi-Yau varieties in positive characteristic, Documenta Mathematica 8 (2003), 97-113.

[69] F. Yobuko, Quasi-Frobenius splitting and lifting of Calabi-Yau varieties in characteristic $p$, Mathematische Zeitschrift 292(1-2) (2019), 307-316.

DOI: https://doi.org/10.1007/s00209-018-2198-7

[70] J.-D. Yu and N. Yui, K3 surfaces of finite height over finite fields, Journal of Mathematics of Kyoto University 48(3) (2008), 499-519.

DOI: https://doi.org/10.1215/kjm/1250271381

[71] D. Wan, Arithmetic mirror symmetry, Pure and Applied Mathematics Quarterly, (Special Issue: In memory of Armand Borel, Part 1 of 3), 1(2) (2005), 369-378.

DOI: http://dx.doi.org/10.4310/PAMQ.2005.v1.n2.a7

[72] D. Wan, Mirror symmetry for zeta functions, Preprint, Available from arXiv:math/0411464.

[73] W. C. Waterhouse, Abelian varieties over finite fields, Annales Scientifiques de l’École Normale Supérieure, 4 Série 2(4) (1969), 521-560. 